\title{
Sustained antibody response to ZIKV infection induced by NS1 protein is accompanied by the progressive appearance of autoreactive antibodies and cross-reactive $B$ cell clones
}

Cecilia B. Cavazzoni ${ }^{1,2}$, Vicente B. T. Bozza ${ }^{1}$, Lucas Tostes ${ }^{1}$, Bruno Maia ${ }^{3}$, Luka Mesin ${ }^{2}$, Ariën Schiepers ${ }^{2}$, Jonatan Ersching ${ }^{2, \dagger}$, Romulo L.S. Neris ${ }^{3}$, Jonas N. Conde ${ }^{4}$, Diego R. Coelho ${ }^{4}$, Luciana Conde ${ }^{1}$, Heitor A. de Paula Neto ${ }^{5}$, Tulio M. Lima ${ }^{6}$, Renata G.F. Alvim ${ }^{6}$, Leda R. Castilho ${ }^{6}$, Ronaldo Mohana-Borges ${ }^{4}$, Iranaia Assunção-Miranda ${ }^{3}$, Alberto Nobrega ${ }^{3}$, Gabriel D. Victora ${ }^{2}$, Andre M. Vale ${ }^{1, *}$

${ }^{1}$ Laboratório de Biologia de Linfócitos, Instituto de Biofísica Carlos Chagas Filho, Universidade Federal do Rio de Janeiro, Rio de Janeiro, RJ, 21941-590, Brazil.

${ }^{2}$ Laboratory of Lymphocyte Dynamics, The Rockefeller University, New York, NY, USA

${ }^{3}$ Instituto de Microbiologia Paulo de Góes, Universidade Federal do Rio de Janeiro, Rio de Janeiro, Brazil.

${ }^{4}$ Laboratório de Genômica Estrutural, Instituto de Biofísica Carlos Chagas Filho, Universidade Federal do Rio de Janeiro, Rio de Janeiro, Brazil.

${ }^{5}$ Laboratório de Alvos Moleculares, Faculdade de Farmácia, Universidade Federal do Rio de Janeiro, Rio de Janeiro, Brazil.

${ }^{6}$ Programa de Engenharia Química, Laboratório de Engenharia de Cultivos Celulares (LECC), COPPE, Universidade Federal do Rio de Janeiro, Rio de Janeiro, 21941-972, Brazil.

${ }^{\dagger}$ In memoriam

*Correspondence to André M. Vale: valeam@biof.ufrj.br

\begin{abstract}
Besides antigen-specific responses to viral antigens, humoral immune response in virus infection can generate polyreactive and autoreactive antibodies. Dengue and Zika virus infections have been linked to antibody-mediated autoimmune disorders including GuillainBarrè syndrome. A unique feature of flaviviruses is the secretion of non-structural protein 1 (NS1) by infected cells. NS1 is highly immunogenic and antibodies targeting NS1 can have both protective and pathogenic roles. In the present study, we investigated the humoral immune response to Zika virus NS1 and found NS1 to be an immunodominant viral antigen associated with the presence of autoreactive antibodies. Through single B cell cultures, we coupled binding assays and BCR sequencing, confirming the immunodominance of NS1. Of note, we demonstrate the presence of self-reactive clones in germinal centers after both infection and immunization, some of which clones presenting cross-reactivity with NS1. Sequence analysis of anti-NS1 B cell clones showed sequence features associated with pathogenic autoreactive antibodies. Our findings demonstrate NS1 immunodominance at the cellular level as well as a potential role for NS1 in ZIKV associated autoimmune manifestations.
\end{abstract}




\section{Introduction}

The protective function of antibodies is instrumental for the control of most viral infections. However, in addition to antigen-specific responses to viral antigens, it has long been noted that viral infection can be accompanied by the appearance of polyreactive and often autoreactive antibodies of unknown function [1]. Emergence of self-reactive immunoglobulins has been reported for multiple viral diseases; these autoreactive antibodies have the potential to lead to autoimmune manifestations, which can be transient or long-lasting (reviewed in [2]). The origin of the stimulus driving autoantibody generation in viral infection remains controversial. On one hand, antigen mimicry between viral and self-antigens may explain the appearance of selected autoantibodies. Alternatively, a role for cytokine storm leading to nonspecific, polyclonal $B$ cell activation followed by disruption of $B$ cell repertoire homeostasis and self-tolerance has also been proposed [3].

Recently, dengue virus (DENV) and zika virus (ZIKV) infections have been linked to the occurrence of autoimmune disorders of vascular, ophthalmic or neurological origin, including Guillain-Barrè syndrome [4, 5], in which autoantibodies seem to play a prominent role [6]. A unique feature of DENV, ZIKV and other flaviviruses is the abundant secretion of the nonstructural protein 1 (NS1) in its hexameric form by infected cells [7-10]. While intracellular NS1 was shown to be necessary for viral replication, its role as an extracellular soluble factor is poorly understood [11]. It has recently been shown that NS1 can lead to endothelial dysfunction [12]. Moreover, work from multiple groups has shown that NS1 is highly immunogenic [13-15]. Notably, antibodies to DENV NS1 can attenuate the outcome of severe dengue and vaccination with ZIKV and DENV NS1 have been shown to be protective in animal models [16-19]. These studies suggest a role for NS1 in the pathogenesis of flavivirus infections, as well as a protective role for anti-NS1 antibodies. However, anti-DENV NS1 antibodies have also been implicated in autoreactivity and may contribute to dengue pathology, possibly through antigen mimicry between NS1 and components of self [20-23]; these observations raise concerns about the safety of NS1 as a vaccine antigen, and further studies are necessary to understand the humoral immune response to this molecule.

Studies of the pathogenesis of human viral infections in animal models face several limitations due to innate viral resistance of mouse species to many human viruses. For this reason, multiple groups have developed models that rely on immunocompromised mice, such as IFNAR-deficient strains, which show greater susceptibility to viral infections [24-27]. Although such strategies are useful in studies of viral pathology, they are less useful for the analysis of humoral immune responses, as type-I interferons broadly influence acquired immunity and directly impact activation of $B$ cells by modulating $B$ cell receptor $(B C R)$ signaling $[28,29]$ possibly also affecting clonal selection and entry into germinal centers (GCs) [30]. For 
these reasons, an immunocompetent mouse model of ZIKV infection is preferred for the study of antibody response. Accordingly, recent studies have used such models to characterize the T cell [31-33] and neutralizing antibody response to ZIKV [34]. Using immunocompetent mouse models, we and others have shown that CD4+ T cells activate a robust IFNYdependent $B$ cell response, which is associated with production of neutralizing IgG2a antibodies that bind to ZIKV envelope proteins including envelope protein domain III (EDIII) and have been associated with virus neutralization. Of note, passive transfer of serum from infected immunocompetent A129 mice protected immunocompromised mice against lethal heterologous challenge with ZIKV [35-37].

In the present study, we investigated the humoral immune response to ZIKV NS1 using an immunocompetent mouse model of ZIKV infection as well as NS1 immunization. The antibody response in infected animals showed that NS1 is an immunodominant viral antigen. Importantly, humoral response to NS1 is associated with the presence of autoreactive antibodies, both after infection or immunization. In-depth analysis of B cell clone selection into GCs, coupling single B cell cultures with BCR sequencing confirmed the strong immunodominance of NS1 and revealed the presence of frequent self-reactive clones among GC B cells, some of which were cross-reacted with NS1. Anti-NS1 B cell clones were enriched in charged amino acid residues in the CDR-H3 region, a feature shared by self-reactive clones $[38,39]$. Anti-NS1 clones also showed low levels of somatic hypermutation (SHM) possibly indicative of adaptation of the germline repertoire to this antigen. The presence of self-reactive $B$ cell clones in GCs, formed in response to an immunodominant viral antigen, strongly supports a break of tolerance at the cellular level. Taken together, these findings indicate the potential relevance of NS1 for ZIKV pathogenicity and its associated autoimmune manifestations.

\section{Results}

\section{Immunocompetent BALB/c mice develop a specific antibody response to ZIKV infection focused on NS1}

To study the humoral immune response to ZIKV infection in immunocompetent mice, we injected young adult BALB/c WT mice intravenously with $10^{7}-10^{8}$ PFU of the Brazilian ZIKV isolate PE243 [40] and followed antibody responses for 50 days post-infection (d.p.i) (Fig. 1 A). Mice showed increased spleen weight from day 7 to 28 after infection (Fig. 1 B), as well as altered total serum immunoglobulin concentrations. Increased serum IgM was detected at 7 d.p.i. (Fig. $1 \mathrm{C}$ ) and total serum IgG concentration increased progressively between 7 and 21 d.p.i., stabilizing at a higher concentration than controls for up to 50 days after infection (Fig. 1 D). 
Serum lgM binding to envelope proteins peaked at 7 d.p.i. (Fig. $1 \mathrm{E}$ ) followed by a peak in IgG at 14 d.p.i., (Fig. 1 F) as detected using Zika virus-like particles (VLPs) that display proteins $E$ and $M$ in their mature form [41]. Domain III of protein $E$ (EDIII) is a target of neutralizing antibodies for different flaviviruses [34, 42-44]. We therefore also assayed serum for IgG binding to this portion of the $E$ protein. We found that EDIII-specific lgG peaked in serum at 14 d.p.i. (Fig. $1 \mathrm{G}$ ), at which point serum neutralizing activity was also observed (data not shown).

We next searched for NS1-binding $\operatorname{lgM}$ and $\lg G$ antibodies in sera of infected mice. NS1 is known to be abundantly secreted into the extracellular milieu by flavivirus-infected cells [45] and is highly immunogenic [8]. IgM binding to NS1 remained almost unchanged compared to uninfected controls (Fig. $1 \mathrm{H}$ ). Interestingly, although serum IgG specific to NS1 appeared later than that targeting envelope proteins, anti-NS1 IgG increased progressively after infection, remaining at very high levels for as long as 50 d.p.i. (Fig. 1 I). Since viral RNA was undetectable in blood and brain tissue, in order to investigate whether our observations were dependent on viral replication leading to secretion of NS1 protein by infected cells, we performed the same experiment with UV-inactivated virus. Mice were injected intravenously with $10^{7}-10^{8} \mathrm{PFU}$ of UV-inactivated (iZIKV) or replicative ZIKV isolate PE243 [40] and evaluated for 60 days after infection (Fig. S1 A). UV-inactivated ZIKV did not induce an increase in spleen weight (Fig. S1 B). Despite the presence of $E$ protein-specific lgG in serum (Fig. S1 C), these immunoglobulins did not target domain III (Fig. S1 D), and NS1-specific IgG was not detected in serum (Fig. S1 E). Taken together, these results demonstrate that, although immunocompetent mice survive ZIKV infection with few or no clinical signs, infection with replicative virus induced a robust humoral immune response, which became progressively dominated by antibodies to the NS1 antigen.

\section{Dominance of NS1-binding IgG in serum correlates with the emergence of autoreactive antibodies}

The increasing levels of anti-NS1 IgG antibodies from 21 d.p.i. onwards prompted us to further investigate the NS1-specific response in infected mice. Serum titration at different time points suggested an increase in concentration or affinity of IgG for the viral antigen (Fig. 2 A). Endpoint titers increased gradually to approximately 200,000 at the latest time point assayed (Fig. 2 B). Serum IgG was predominantly of the IgG2a isotype throughout infection, as expected for anti-viral responses [46]. Of note, there was a late contribution of IgG1 to total serum IgG titer (Fig. $2 \mathrm{C}$ and D). Given that gamma 1 constant region gene is located upstream of gamma $2 a$, ruling out sequential switching between these isotypes, our data suggest continued engagement of $B$ cell clones after the initial phase of the response. We also assessed the binding of IgG to closely-related dengue virus antigens. As observed in human 
antibody response to ZIKV infection [15, 47], we found cross-reactivity between ZIKV EDIII and DENV EDIII (Fig. 2 E; Fig. S2, A and B), whereas ZIKV NS1-specific IgG did not crossreact with DENV NS1 protein (Fig. 2 F; Fig. S2, C and D).

In addition to the presence of virus-specific antibody responses, viral infections are often associated with hyperglobulinemia due to non-specific polyclonal activation of B lymphocytes [1]. To assess the potential of ZIKV infection to induce a polyclonal, non-specific humoral immune response, we looked for lgG binding to unrelated antigens such as heat shock proteins from both mammalian and commensal bacterial origins, which are commonly targeted by autoantibodies in different systems [48-50] (Fig. S2, E and F). IgG binding to unrelated antigens, possibly due to polyreactivity, was present at early time points and rapidly decayed, following the kinetics of antibody response to viral structural proteins shown in Figure 1. Interestingly, serum IgG from ZIKV-infected mice also displayed widespread binding to selfantigens, as revealed using a semiquantitative immunoblot assay that enables global analysis of the self-reactivity of antibodies present in serum [51, 52]. Accordingly, at 14 d.p.i., ZIKVinfected mice exhibited serum IgG reactivity to multiple self-antigens from syngeneic brain and muscle tissues (Fig. $2 \mathrm{G}$ ), suggesting a break in self-tolerance during the early humoral immune response.

Polyclonal B cell activation and non-specific polyreactive responses are mostly present in the acute phase of the immune response to viral infections, and do not contribute meaningfully to serum IgG titers at later time points when infection subsides. These observations prompted us to evaluate whether self-antigen reactivity declines at later time points after infection. Strikingly, serum IgG self-reactivity was progressively stronger at later time points (Fig. 2, GI). Of note, although different mice shared several reactivities towards antigens with similar migration patterns (Fig. 2, G and H), individual immunoreactivity profiles did not necessarily converge in time towards a unique reactivity profile (Fig. $2 \mathrm{~J}$ ).

To further investigate the progressive increase of self-reactive IgG following infection, we tested serum samples from an additional cohort of ZIKV-infected mice for binding to a HEp-2 cell line extract. These cells are frequently used in standard clinical assays for anti-nuclear antibodies (ANAs), but also as a source of cytoplasmic self-antigens [38]. Consistently, a broad range of immunoreactivities arose over time after infection (Fig. $2 \mathrm{~K}$ ). The levels of IgG reactive to a $60 \mathrm{kDa}$ self-antigen paralleled those of NS1-specific, but not of EDIII-specific, lgG over time (Fig. 2 L). The overlapping kinetics of anti-NS1 response and self-reactive serum lgG led us to hypothesize there could be a link between the maintenance of autoreactive antibodies and a dominant and sustained anti-NS1 antibody response. 


\section{Virus-specific and autoreactive B cell clones are present in GCs after ZIKV infection}

Viral infections typically induce T-dependent antibody responses, in which follicular B cells (FO) enter GC reactions where they undergo clonal expansion, SHM, and selection, leading to antibody affinity maturation (reviewed in [53]). After intravenous (i.v.) ZIKV infection, we observed abundant GC formation in spleen. Frequencies of different splenic B cell populations were found to be altered; the reduction in frequency of FO B cells likely reflected GC formation and correlated with levels of serum IgG specific to ZIKV proteins. GC B cell frequency peaked at day 14 after infection and started to decrease by day 21 , receding to background levels at 28 d.p.i. (Fig. 3 A).

To investigate the possible link between the antibody response to NS1 and the presence of self-reactive lgG in serum, we devised an experimental strategy that allowed us to more appropriately compare GC B cells between infected and immunized mice. For that purpose, mice were subcutaneously infected in the footpad with ZIKV and draining lymph nodes (LN) were collected on different days post-infection to isolate GC B cells (Fig. 3 B). Consistent with our previous results, we found increasing levels of IgG binding to ZIKV NS1 in serum up to 45 days after subcutaneous infection. This increase was not observed when the same amount of UV-inactivated virus (iZIKV) was injected, indicating that this phenomenon requires replicative ZIKV infection (Fig. $3 \mathrm{C}$ ). We could also observe GC formation in LN after both ZIKV infection and iZIKV immunization, although the latter were both of lower magnitude and shorter in duration (Fig. 3, D and E). At 14 d.p.i., corresponding to the peak of the response, we isolated and cultured GC B cells for Ig production in vitro. GC B cell cultures were performed as described by Kuraoka et al.[54], with modifications, including not adding IL-4 to prevent in vitro class switching. As a result, the proportions of IgG isotypes found in culture supernatants broadly matched those of ZIKV-specific antibodies in serum (Fig. S3).

Using limiting dilution analysis (LDA), we were able to estimate the frequency of GC B cells secreting immunoglobulins binding to VLP or NS1 (Fig. 3, F and G), as well as to selfantigens (Fig. $3 \mathrm{H}$ ). Quantification of the number of responding $B$ cell clones per culture ensures accurate determination of the frequency of GC B cells reactive to a given antigen $[55$, 56]. GC B cells from mock-, iZIKV-, and ZIKV-injected mice showed similar frequencies of response to polyclonal LPS stimulus, with $30 \%$ to $50 \%$ of GC B cells proliferating and differentiating into lgG-secreting plasma cells in all conditions (Fig. S3 A, and data not shown). At 14 d.p.i., no reactivity to viral surface antigens was detected in GC B cells derived from control mice (mock) and less than $0.1 \%$ of GC B cells from iZIKV-imumunized mice secreted IgG that bound detectably to VLP. Moreover, we were unable to detect GC B cell clones secreting NS1-reactive IgG in neither mock- nor iZIKV-immunized mice (data not shown). On the other hand, in ZIKV-infected mice, GC B cells specific for NS1 were readily detected at 
relatively high frequencies (Fig. 3 F). The proportion of GC B cells reacting to NS1 (4.5\%) was 10 -fold higher than that of GC B cells binding to envelope proteins $(0.4 \%)$ (Fig. $3 \mathrm{G}$ ) and correlated with virus-specific lgG levels observed in serum (Fig. 3 C; see also Fig. 1, F and J). These findings underscore the extent of the immunodominance of NS1 over envelope antigens also at the cellular level.

We then performed the global analysis of self-reactivities, as used for serum IgG (Fig. 2 $H$ ), with GC B cell culture supernatants. By combining LDA and immunoblot assays, we were able to estimate the frequency of cell secreting IgG binding to self-antigens in each $G C B$ cell culture (Fig. $3 \mathrm{H}$ ). Our analysis showed that autoreactive B cells were present within GCs formed after exposure to replicative ZIKV, whereas these were virtually absent upon exposure to UV-inactivated virus (Fig. $3 \mathrm{H}$ ). Importantly, the self-reactivity found with highest frequency (Ag2) accounted for $1 \%$ of GC B cells in the lymph node (Fig. $3 \mathrm{H}$ ), at least twice the frequency of GC B cells detectably specific for virus envelope proteins (Fig. $3 \mathrm{G}$ ). These data suggest a role for NS1 in triggering the autoreactive antibody response following ZIKV infection.

\section{ZIKV NS1 immunization recruits a high frequency of NS1-specific B cells to the GC}

To directly test whether an anti-NS1 humoral immune response generates autoreactive antibodies, we immunized mice in the footpads with purified recombinant ZIKV NS1 in the presence of a TLR7 agonist (R848). For comparison, two other groups were included. Mice were immunized with ZIKV virus-like particles (VLPs), displaying envelope proteins ( $E$ and $M$ ) in the presence of R848 or with a combination of VLPs and NS1 (Fig. 4 A). We first analyzed the humoral immune response against the viral antigens. We found that, from day 14 postimmunization onwards, serum levels of NS1-specific IgG were higher than those of VLPspecific IgG in both combinations (Fig. 4 B). Despite the similar frequencies of total GC B cells in popliteal LNs (Fig. 4 C), frequencies of specific B cells within the GC varied. At day 14 after immunization with VLP/R848, the frequency of GC B cells binding to VLPs was $2.6 \%$ (Fig. 5 $E$, left panels). After immunization with NS1/R848, however, frequency of specific GC B cells was approximately 10 -fold higher, at $27 \%$ (Fig. $4 \mathrm{E}$, right panels). Compared to the single antigen immunization protocol, VLP and NS1 in combination reduced the frequency of GC B cells specific for both antigens to $1.2 \%$ and $3 \%$, respectively. (Fig. $4 \mathrm{E}$, middle panels). This reduction was not due to lower secretion of $\lg$ in vitro, since similar levels of NS1 specific IgG were found in culture supernatants, irrespective of the presence of VLPs in the immunization (Fig. 4 F). Altogether, antigen-specific GC B cell frequency and kinetics in immunized mice mirrored the virus-specific lgG levels found in the ZIKV infection model, corroborating the observed immunodominance of NS1 over ZIKV envelope antigens.

\section{Paucity of somatic hypermutations in expanded GC B cell clones from ZIKV NS1- immunized mice}


To further characterize the B cell response to ZIKV NS1, we sorted single GC B cells from popliteal LNs of mice immunized with this antigen at different time points and performed Igh sequencing. To gain insight into specific features of ZIKV NS1 B cell response, we also immunized mice with DENV NS1 for comparison, since, even though the two proteins are structurally homologous, antibodies generated after infection with ZIKV did not cross-react with DENV NS1 (see Fig. 2 F). As expected, GCs found in popliteal LNs of mice immunized with either ZIKV or DENV NS1 proteins 10 days post-immunization were highly clonally diverse, whereas clones with increased frequency accumulated over time (Fig. 5A).

Analysis of $\mathrm{V}_{H}$ segment usage showed preferential use of the $\mathrm{V}_{H} 1$ (J558) gene family, irrespective of immunizing antigen and time point analyzed (Fig. S4 A). The average number of mutations in $V_{H}$ gene segments was similar for both antigens and increased over time as one would expect. However, interestingly, $V_{H}$ mutation numbers were lower among clones found more frequently (likely those undergoing positive selection) in GCs formed after ZIKV NS1 immunization when compared to those formed after DENV NS1 immunization (Fig. 5 B). In contrast to those clones from ZIKV NS1 immunization, B cells in GCs from DENV NS1immunized mice tended to progressively accumulate mutations in more expanded clones over time (Fig. 5 C).

Although no significant differences in CDR-H3 length were found in GC B cells after DENV or ZIKV NS1 immunization at any time point (Fig. S4 B), there was a preference for 11-amino acid-long CDR-H3s in both ZIKV NS1 and DENV NS1 immunized mice when compared to the naïve B cell repertoire (Fig. S4 C). Average CDR-H3 hydrophobicity tended to be lower for ZIKV NS1 than for DENV NS1 (Fig. 5 D). The distribution of CDR-H3 average hydrophobicity revealed few highly charged sequences among $B$ cells responding to ZIKV NS1 antigen (Fig. $5 \mathrm{E})$. Comparison of the amino acid composition of $\mathrm{CDR}-\mathrm{H} 3$ regions of clones obtained in response to both antigens at different time points revealed an enrichment over time for charged amino acids, especially arginine after ZIKV NS1 immunization (Fig. 5 F). Of note, charged amino acids in the antigen binding sites of $\operatorname{lgG}$ are often critical for self-reactivity [57]. However, a marked glycine (neutral amino acid) enrichment was also observed, which could counterbalance the charged amino acid bias, resulting in a moderate change in average hydrophobicity in CDR-H3 sequences as shown in Fig. $5 \mathrm{D}$. Evaluating the percent difference between hydrophobic and charged amino acids in CDR-H3 sequences, we found that $\mathrm{B}$ cell clones derived from the ZIVK NS1 immunized animals used charged amino acids more frequently than hydrophobic ones when compared to the DENV NS1-immunized mice (Fig. 5 $\mathrm{G).}$

The recruitment of $B$ cells enriched in charged CDR-H3 sequences into the GCs upon immunization with ZIKV NS1 could be related to the emergence of autoreactive lgG. Although, 
the specificities of the immunoglobulins encoded by these sequences are not known, results obtained with GC B cells cultures suggest that most of these cells (63\% for ZIKV NS1 and 97\% for ZIKV VLP) do not secrete antibodies with detectable binding to the antigen used in immunization (see Fig. $4 \mathrm{E}$ ). One might expect that the most expanded clones would be those with the greatest affinity for the antigen; however, it remains possible that the most mutated clones would become expanded only at later time points, after more extensive selection, and would therefore not be detected at high frequencies in the samples we analyzed. To better understand this phenomenon, we sought to correlate the features of immunoglobulin variable gene sequences with the specificities of their secreted antibodies.

\section{ZIKV NS1 immunization recruits into GCs B cell clones enriched for charged amino acids in the CDR-H3s and self-reactivity}

To understand the relationship between Ig sequence features and antigen specificity, we used single GC B cell cultures, which allow the assessment of both Ig sequence and binding properties from the same cell (adapted from [56]). For this purpose, GC B cells were harvested from popliteal LNs of mice immunized with ZIKV or DENV-NS1 protein at different time points after immunization. After 7 days in culture, cells were processed for lgh sequencing and supernatants were used for specificity assessment. We first determined the frequency of GC B cells secreting ZIKV NS1-binding IgG. A total of 271 monoclonal antibodies were tested for binding to NS1 protein. In line with the rising levels of serum IgG specific for NS1 (shown in Fig. 4 B), frequency of ZIKV NS1-specific B cells also increased over time, from an average of $30 \%$ at 10 d.p.i. to $50 \%$ at 21 d.p.i. (Fig. 6 A). We then divided B cell clones into NS1binders and non-binders for further analyses. Clonal expansion was evident among NS1binding GC B cells, peaking on day 14 after immunization (when almost $60 \%$ of NS1-binders were found in detectably expanded clonotypes), consistent with antigen-driven selection (Fig. 6 B). At 21 d.p.i., the fraction of expanded clones among NS1-binders decayed to a frequency similar to that found at day 10 post-immunization. Although the number of clones analyzed at this time point is limited, this result could suggest continued ingress of new B cell clones into ongoing GCs (Fig. 6 B). In contrast, non-binder clones showed significantly less clonal expansion at all time points analyzed (Fig. 6 B). Interestingly, and consistent with data shown previously (Fig. $5 \mathrm{~B}$ ), we did not find evidence for positive selection of NS1-binding clones bearing large numbers of somatic mutations (Fig. $6 \mathrm{C}$ ).

We then investigated the physicochemical features of the CDR-H3 sequences expressed by B cells recruited to GCs after ZIKV NS1 immunization. On day 14 p.i., both ZIKV NS1 binders and non-binders showed similar CDR-H3 hydrophobicity, whereas on day 21 p.i., ZIKV NS1 binders tended to be less charged and closer to neutrality than non-binders, an effect just short of statistical significance (Fig. 6 D). To examine whether NS1 binding 
correlated with the abundance of positively charged amino acids in CDR-H3, we plotted the anti-NS1 reactivity per clone at each time point, highlighting CDR-H3s expressing three or more charged amino acids (Fig. $6 \mathrm{E}$, red dots). CDR-H3 sequences bearing charged amino acids were frequent and evenly distributed among NS1-binder and non-binder clones, accounting for $25 \%$ to $35 \%$ of $\mathrm{CDR}-\mathrm{H} 3$ sequences (Fig. $6 \mathrm{E}$ ). Overall, CDR-H3 sequences of GC B cells from ZIKV NS1 immunized mice were enriched for charged amino acids (including arginine), as compared to what has been observed in the naïve repertoire [58] as well as in comparison to clones from DENV NS1 immunized mice (Fig. 5 F and G; Fig. 6 E).

The presence of positively charged amino acids within CDR-H3 is a common feature associated with self-reactivity [59]. To determine whether particular Igh sequence features were correlated with self-reactivity, we tested 58 monoclonal antibodies for binding to autologous muscle and brain extracts (Table S1 and Fig. 6 F). Corroborating the results obtained with serum and limiting dilution of GC B cells, no self-reactivity was found among GC $B$ cells from mice immunized with DENV NS1 at any time point (Table S1 and Fig. 6 F). In contrast, $18.2 \%$ of evaluated single cells obtained at day 14 after immunization with ZIKV NS1 were self-reactive. This frequency increased to $42.3 \%$ of the analyzed cells from 21 d.p.i. Highly charged CDR-H3 were not determinant for self-reactivity since we found this feature among both self-reactive and non-self-reactive clones. Nevertheless, we did find a handful of clonotypes bearing hydrophobic amino acids in their CDR-H3 that were predominantly selfreactive (Table S1; Fig. 6 , F and I).

\section{GC B cells from ZNS1 immunized mice display widespread self-reactivity irrespective of clonal sizes, SHM and nominal antigen-selection}

We further analyzed the SHM patterns and clonal frequencies of $58 \mathrm{GC}$ B cells for which we obtained complete data set, correlating it with IgG reactivity to NS1 and to self-antigens. Among GC B cells obtained at day 14 after immunization with ZIKV NS1, we found cells with similar CDR-H3 but different SHM patterns, including a clonal family bearing a "glycineenriched CDR-H3" (ARGGGYDGFAY). The least mutated cell in this group was inferred to have acquired a non-silent mutation in the D2-02 gene segment, which led to the replacement of a tyrosine by a phenylalanine in the CDR-H3 (becoming ARGGGFDGFAY). The germline D2-02 D gene segment encodes the GYD motif, whereas the mutated version encoded a GFD motif, which displayed increased poly-reactivity against self-antigens together with increased reactivity to ZNS1 (Fig. S5 A and Table S1). The two clones bearing the glycine-enriched CDR-H3 that bound NS1 and cross-reacted with self-antigens were also highly expanded, suggesting that antigen-specific GC B cells - whether self-reactive or not - are capable of undergoing positive selection (Fig. $6 \mathrm{G}$, green dots). 
At 21 d.p.i., other groups of related clonotypes were found with different SHM patterns (Table S1 and Fig. 6 F). An interesting autoreactive clone carried the ARGTLYAMDY CDRH3 (Fig. $6 \mathrm{~F}$, highlighted in blue colors). In addition to being among the most hydrophobic sequences in our list, we found more mutated variants both non-autoreactive (ARGTLYIMDY)

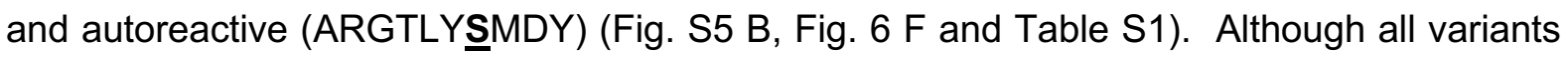
of this clonal family bound to ZIKV NS1 protein, the most mutated one (ARGTLY $\underline{S} M D Y$ ) displayed the highest relative reactivity to NS1 (Table S1) as well as increased poly- and selfreactivity (Fig. S5 B and Fig. 6 F, highlighted in light blue). In this case, only the least mutated sequence (ARGTLYAMDY) was found more than once in the data set. Notably, the two most prominent self-reactivities found in the ARGTLY $\underline{S} M D Y$ clone were also found in serum IgG from mice infected with ZIKV 35 d.p.i. (Fig. S5 B and Fig. 6 F, blue arrows).

Overall, ZIKV NS1 clonotypes found at the highest frequencies (> 5 counts) were generally non-self-reactive (Fig. $6 \mathrm{G}$ ). Moreover, all NS1-binding immunoglobulins tested for selfreactivity, regardless of their self-reactivity status, had numbers of $V_{H}$ mutations that were similar and higher than those of non-antigen-specific cells, again suggesting antigen-driven selection (Fig. $6 \mathrm{H}$ ). The most mutated clones did not bind to self-antigens and were found exclusively as singletons, irrespective of binding to ZIKV NS1 (Fig. $6 \mathrm{G}$ and H, gray dots).

The results obtained with single cell sequencing and binding assays were summarized in Figure $6 \mathrm{I}$. Clonality scores showed that the presence of more expanded clones was a property of earlier GCs, obtained at day 14 post-infection, whereas late GCs were enriched in smaller clones. Interestingly, the decrease in clonal dominance inversely correlated with increased self-reactivity, suggesting a replacement of early clones by a new wave of B cells with selfreactive potential. SHM appeared not to increase from day 14 to 21 , which would be in line with clonal replacement. Self-reactive clones were already present at day 14 , all of which exhibited cross-reactivity with ZIKV NS1. It is noteworthy that autoreactive clones with no cross-reactivity with NS1 were present at day 21 but not at day 14 p.i.. Although the number of clones assayed for autoreactivity limits the strength of this conclusion, we found more selfreactivity among NS1-binders than among non-binders. Collectively, our data support a role for NS1 in recruiting cross-reactive B cell clones into GCs, some of which are self-reactive clonotypes showing evidence for clonal expansion and paucity of VH gene mutations.

\section{Discussion}

The humoral immune response to ZIKV infection in humans is characterized by early appearance of antibodies to structural proteins of the viral envelope followed by a later increase in antibodies to the non-structural protein NS1 [14, 15]. Here, we found that the humoral immune response of immunocompetent BALB/c mice to ZIKV infection follows a 
similar pattern. The BALB/c antibody response is characterized by early emergence of envelope-specific IgG, including EDIII-specific and neutralizing antibodies, followed by a delayed response dominated by antibodies to NS1. The delayed response to NS1 may be a consequence of its absence from the viral particle, possibly explaining the initial dominance of anti-envelope antibodies. Only after productive ZIKV infection do cells start producing and secreting large amounts of NS1, which then accumulates in bodily fluids and on the surface of infected cells. NS1 circulates in blood, and has been implicated both in damage to endothelial cells and in immune evasion through inhibition of the complement cascade [12, $23,60,61]$. Of note, antibodies to NS1 have been shown to be protective against Zika and Dengue diseases, and immunization with NS1 has been considered as a possible prophylactic strategy $[18,19,62]$.

Antibodies to DENV NS1 have also been implicated in pathological autoreactivity in humans $[20,63]$. From this perspective, it is interesting to note that reactivity towards selfantigens present in different tissues or cell extracts was observed in the humoral immune response of BALB/c mice to ZIKV infection. The presence of autoreactive antibodies in viral diseases is not an uncommon finding and is often attributed to non-specific polyclonal $B$ cell activation. Autoreactive B cells could be stimulated in a T-independent manner, via simultaneous TLRs and BCR signaling both in GCs and in extrafollicular foci [64-67]. However, polyclonal $B$ lymphocyte activation is mostly an acute phase phenomenon that subsides with clearance of the virus. Here, by contrast, we found that self-reactivity was sustained and even augmented at later time points after infection, long after ZIKV had been eliminated. Interestingly, NS1-specific antibodies were also long-lasting in serum and correlated well with the kinetics of appearance and maintenance of autoreactive antibodies in BALB/C mice. Serum IgG isotypes composition of the NS1 specific repertoire was coherent with the sustained IgG anti-NS1 response and with engagement of new clonotypes. Early predominant IgG2a response was followed by a later emergence of IgG1, while lgG2a titers still increased up to 50 days after infection. As previously observed, gamma 1 constant region gene is located upstream of gamma $2 a$, ruling out sequential switching between these isotypes, implying the recruitment of new clones to sustain anti-NS1 antibody levels. It is still not clear whether the response to the viral antigen is maintained by self-antigens or by the viral antigen itself, that could be present at later time points, even though the virus was not detected after the first week of infection, as long-term antigen persistence in follicular dendritic cells has been well documented [68]. Hence, kinetics of the NS1 response is unique and differs from that induced by viral surface antigens, progressively dominating the humoral response. The shift from antienvelope to anti-NS1 response could be a viral escape strategy, since neutralization is achieved mostly by antibodies directed to viral surface proteins. 
Our analysis of GC B cells from ZIKV infected mice revealed both virus-specific and selfreactive $B$ cells. Using single-cell cultures of GC B cells from mice immunized with ZIKV NS1, we showed that both anti-NS1 and autoreactivity could often be attributed to the same cell. Although GC responses to ZIKV NS1 and DENV NS1 did not differ in kinetics and were of short duration, autoreactive clones were found at all time points investigated after ZIKV NS1 immunization but not after DENV NS1 immunization. It is worth noting that serum IgG antiZIKV NS1 did not cross react with DENV NS1, in agreement with the distinct reactivity profiles reported here.

Self-reactive antibodies associated with autoimmune diseases, such as lupus, have been shown to be enriched for charged amino acids, especially arginine, in CDR-H3 [39]. Under normal circumstances, immunocompetent $B A L B / c$ mice are considered resistant to production of these autoantibodies [69,70]. This is in part due to the low prevalence (around 5\%) of arginine in the $\mathrm{CDR}-\mathrm{H} 3$ region of the $\mathrm{BCR}$ s of mature recirculating $\mathrm{B}$ cells in $\mathrm{BALB} / \mathrm{c}$ mice [58]. Half of the arginines in CDR-H3 regions of immature $B$ cells in BALB/c mice derive from $N$ additions and half from germline $D_{H}$ sequences [70]. Here, we found an enrichment for charged amino acids, including arginine, in CDR-H3 of GC B cell receptors after ZIKV NS1 immunization, as compared to DENV NS1. Interestingly, one particular clone found at high frequency among GC B cells at day 14 after immunization with ZIKV NS1 utilized the D gene segment DSP2.11 (D2-14), the only one to encode arginine in the germline sequence in reading frame 1 [70]. Notably, a marked glycine enrichment in CDR-H3 was also observed, which might contribute to maintaining the average hydrophobicity close to neutrality. NS1 is a complex multifunctional protein that forms a peculiar hydrophobic core in its hexameric structure which is secreted by infected cells [71]. Interestingly, in addition to the enrichment for charged amino acids, we also found self-reactive clonotypes bearing hydrophobic CDR$\mathrm{H} 3 \mathrm{~s}$; whether these particular clonotypes are able to bind to hydrophobic epitopes on NS1 protein, revealing potential immunogenicity of this hydrophobic core remains to be determined.

Igh sequencing of B cells isolated from GCs of ZIKV NS1 or DENV NS1-immunized mice showed similar usage of $\mathrm{V}_{\mathrm{H}}$ segments and similar average number of $\mathrm{V}_{\mathrm{H}}$ mutations, increasing over time as one would expect. However, after ZIKV NS1 immunization, $\mathrm{V}_{\mathrm{H}}$ mutation numbers were lower among clones found more frequently, possibly indicating selection of neargermline $\mathrm{B}$ cell clones. We speculate that this could be due to the availability, in the preimmune repertoire, of B cells able to bind to ZIKV NS1 protein with enough affinity to differentiate rapidly into plasma cells before accumulating many mutations. As recently shown by Burnett and colleagues, selection in GCs is skewed towards lower affinity for self-antigens prior to increasing affinity to foreign antigens [72]. In this context, it is possible that anti-ZIKV NS1 clones could generate plasma cells before acquiring enough mutations to diminish affinity to self-antigens. While it is known that plasma cell differentiation in GCs is dependent on high 
BCR affinity, the mechanisms through which the affinity threshold for differentiation is set are unclear $[73,74]$.

Coupling sequence analysis to binding assays, we found NS1-specific B cells that had similar numbers of $\mathrm{V}_{\mathrm{H}}$ mutations (higher than those of non-specific clones), regardless of being self-reactive or not. By contrast, self-reactive B cells that did not bind to ZIKV NS1 protein were the least mutated of all and were also not detectably expanded (Figure $6 \mathrm{G}$ and $\mathrm{H}$ ). These data suggested the presence of antigen-driven selection in spite of self-reactivity, although, among NS1-specific cells, self-reactive cells seemed disfavored as compared to non-self-reactive ones. For instance, the glycine-enriched CDR-H3 ARGGGYDGFAY likely further mutated to ARGGGFDGFAY, generating increased poly- and self-reactivity while simultaneously increasing its reactivity to ZNS1. The autoreactive clonotype ARGTLYAMDY, on the other hand, was found in further mutated versions, ARGTLYIMDY, which lost autoreactivity, and ARGTLY $\underline{S} M D Y$, which remained autoreactive (Fig. S5 A and Table S1). Overall, our observations suggest that GC B cell clones undergoing selection after ZIKV NS1 immunization tend to be closer to germline than those in DENV NS1 immunization, an intriguing finding that requires further investigation.

In conclusion, we show here, for the first time, that ZIKV NS1-specific GC B cells can cross-react with self-antigens, possibly by molecular mimicry between ZIKV NS1 and selfantigens, raising the question whether self-antigens can participate in the stimulation of antiNS1 B cell clones. This hypothesis could explain the sustained progression of the anti-NS1 humoral immune response we observed in infected mice, which display similar kinetics to that of self-reactive antibodies in serum. Notably, autoreactive clones that did not react with ZIKV NS1 were also found in GCs, raising the possibility of a "true" break of self-tolerance in the immune response to viral infection that goes beyond antigen mimicry. Finally, the flavivirus NS1 protein has been proven highly immunogenic in humans and capable of inducing protective antibodies and therefore suggested as a potential vaccine antigen $[16,18]$ or therapeutic antibody target [62]. In this context, the data presented here raise concerns about the safety of that approach and call attention to in-depth analysis of B cell clones engaged in response to this viral antigen, especially in its autoreactive component. This concern is echoed in very recent studies highlighting the self-reactive potential of close-to-germline encoded antiviral antibodies to severe acute respiratory syndrome coronavirus 2 (SARS-CoV-2), the infectious agent of coronavirus disease 2019 (COVID-19) [75-78], arguing that the phenomenon we describe may be of importance well beyond the specific case of ZIVK infection. 


\section{Material and methods}

\section{Mice and treatments}

BALB/c adult female mice, aged 6 to 8 weeks, were obtained from NAL-UFF, LAT-UFRJ or The Jackson Laboratory. Mice were kept in a $12 \mathrm{~h}$ light/dark cycle with ad libitum access to food and water. Mice were infected with $10^{6}-10^{7}$ PFU of ZIKV PE243 [40] i.v. and blood samples were collected on days $2,7,14,21,28,35,42,50$ or 60 , as indicated for each experiment. ZIKV strain PE243 (Brazil/South America, gene bank accession no. KX197192) was propagated and titrated in Vero cells and UV-inactivated as previously described [40]. All animal procedures were approved by the Institutional Animal Care and Use Committee of the Centro de Ciências da Saúde da Universidade Federal do Rio de Janeiro and the Rockefeller University.

\section{ELISA for immunoglobulin quantification}

Total IgG and IgM concentration in serum and cell culture supernatants were determined by ELISA as previously described in [79] using anti-mouse IgM and IgG-specific reagents (Southern Biotechnology). Briefly, 96 well plates (Costar) were incubated with anti-lgM or antilgG capturing antibody at $1 \mathrm{ug} / \mathrm{ml}$ (Southern Biotech) and incubated at $4^{\circ} \mathrm{C}$ for $18 \mathrm{~h}$. Then, after 1h of blocking (PBS-1\%BSA), serum samples and culture supernatants were diluted in PBSBSA $1 \%$ by serial dilution starting at $1: 40$ for serum and undiluted for supernatants. Standard curves of polyclonal IgM or IgG were obtained by serial dilution 3-fold for IgM and 5-fold for IgG, starting at $1 \mathrm{ug} / \mathrm{mL}$ for supernatants and $2 \mathrm{ug} / \mathrm{mL}$ for serum samples. Secondary antibodies conjugated to HRP (Southern Biotech) were diluted 1:2000 for IgM and 1:8000 for IgG in PBS-BSA 1\%. After wash with PBS, reactions were developed with TMB substrate solution (Sigma). The reaction was stopped with $\mathrm{HCL} 1 \mathrm{~N}$.

\section{ELISA for antigen-specific immunoglobulin detection}

ELISAs to determine serum levels of anti-VLPs, anti-EDIII and anti-NS1 antibodies as well as specificity of IgG in B cell culture supernatants were performed as previously described[35]. Briefly, 96 well plates (Costar) were coated with peptide (EDIII) $(10 \mu \mathrm{g} / \mathrm{ml})$ or protein $(1 \mu \mathrm{g} / \mathrm{ml})$ diluted in PBS and incubated at $4^{\circ} \mathrm{C}$ for $18 \mathrm{~h}$. Serum samples were diluted following serial dilution $1: 3$ for $\operatorname{lgM}$ and $\lg$ starting with $1: 40$ in the first well. ZIKV and DENV EDIII recombinant proteins were kindly provided by Dr. Orlando Ferreira, IB, UFRJ. VLPs were produced and purified as previously described [41]. ZIKV and DENV NS1 proteins were produced and purified as reported previously.

\section{Immunoblot}

BALB/c tissues (brain and skeletal muscle) were dissociated by Polytron homogenizer (4000 rpm) in homogenizing buffer (Tris- $\mathrm{HCl} 0,5 \mathrm{M} \mathrm{pH} \mathrm{6,8,} \mathrm{SDS} \mathrm{10 \% ,} \mathrm{Mili-Q} \mathrm{water)} \mathrm{as}$ 
described by Haury et al.[52]. Tissue extracts were fractioned by electrophoresis in $10 \%$ polyacrylamide gel under denaturing conditions, at $50 \mathrm{~mA}$ until $6 \mathrm{~cm}$ of migration. Proteins were transferred from the gel to a nitrocellulose membrane by a semi-dry electro transfer (Semi-Dry Electroblotter $B$ ) for 60 minutes at $0,8 \mathrm{~mA} / \mathrm{cm}^{2}$. After transfer, the membrane was kept in $50 \mathrm{~mL}$ of PBS/Tween 20 (BioRad) at 0,2\% vol/vol shaking for 18 hours at room temperature.

Incubation of the membrane with cell culture supernatants or serum samples was performed in a Miniblot System Cassette (Immunetics Inc.) which allows the simultaneous incubation of 28 different samples in separated channels. Supernatant samples were diluted 1:2 and serum samples were diluted 1:100. After wash, membranes were incubated with secondary antibodies conjugated to alkaline phosphatase anti-IgM or anti-IgG diluted 1:2000 (rabbit anti-mouse IgM, Jackson ImmunoResearch, goat anti-mouse IgG, SouthernBiotech). Substrate NBT/BCIP (Promega) was added after wash. Reaction was developed shaking at room temperature and stopped with Milli-Q water. Colloidal gold staining was performed after scanning membranes.

\section{Flow cytometry and cell sorting}

Cells were harvested from spleen, peritoneal cavity and popliteal lymph nodes for flow cytometry and cell sorting. Splenocytes were homogenized with complete RPMI 1640 medium (GIBCO), followed by red blood cell lysis in $1 \mathrm{~mL}$ of ACK lysing buffer (GIBCO) for $1 \mathrm{~min}$ on ice. Single cell suspensions were prepared from the peritoneal cavity lavage with $5 \mathrm{ml}$ of complete RPMI 1640 medium (GIBCO). Popliteal lymph nodes were homogenized with complete RPMI 1640 medium (GIBCO). Cells were washed and resuspended in an appropriate volume for counting and staining. Cells were stained with the following monoclonal Abs conjugated to fluorochromes: anti-B220, CD38, CD138, GL-7, CD21, CD23, CD93 (AA4.1), CD11b, CD5, IgM and CD44 (eBioscience) for 30 minutes at $4^{\circ} \mathrm{C}$ in FACS staining buffer (PBS 1x with 5\% FCS). Analysis and cell sort were then performed on a MoFlo instrument (Dako-Cytomation). B cell populations were defined as follows: GC (B220+ CD138CD38lo/- GL-7+) and FO (B220+ CD138- CD21lo/neg CD23+ GL-7-). Cells were collected directly in sterile tubes containing supplemented OptiMEM (GIBCO) for cell culture.

\section{GC B cell culture in limiting dilution assay (LDA)}

Decreasing number of GC B cells were cultured in $250 \mu \mathrm{l}$ of OptiMEM (GIBCO) supplemented with 10\% heat-inactivated FBS (GIBCO), $2 \mathrm{mM}$ L-glutamine, $1 \mathrm{mM}$ sodium pyruvate, $50 \mu \mathrm{M} 2-\mathrm{ME}, 100 \mathrm{U}$ penicillin, and $100 \mu \mathrm{g} / \mathrm{ml}$ streptomycin. All cultures were performed in 96-well flat bottom plates containing $3 \times 10^{3} \mathrm{NB} 40 \mathrm{~L}$ feeder cells/well as previously described protocol with minor modifications [80]. GC B cells were added starting from 3000 cells/well to 1 cell/well through 3 -fold dilution steps in the presence of $30 \mu \mathrm{g} / \mathrm{ml}$ of LPS 
(Salmonella typhimurium, Sigma-Aldrich) and $2 \mathrm{ng} / \mathrm{ml}$ of IL-21 (Peprotech). After 7 days, cultures were screened by ELISA to determine the frequency of IgG secreting GC B cells according to the Poisson distribution [81, 82].

\section{Single GC B cell cultures}

Single GC B cells were sorted into 96-well round-bottom plates containing $3 \times 10^{3}$ NB21 cells/well as previously described with minor modifications, mostly not adding IL-4 [54]. Supernatants were collected after 7 days of culture and cells were frozen in TCL lysis buffer supplemented with $1 \% \beta$-mercaptoethanol for Igh sequencing.

\section{Igh sequencing}

Single GC B cells from popliteal lymph nodes of Balb/c mice immunized with ZIKV or DENV NS1 proteins were sorted into 96-well PCR plates directly or after 7 days in culture. Plates contained $5 \mu$ l of TCL lysis buffer (Qiagen) supplemented with $1 \% \beta$-mercaptoethanol. RNA extraction was performed using SPRI bead as described in [73]. RNA was reverse transcribed into cDNA using an oligo (dT) primer. Igh transcripts were amplified as described in [83]. PCR products were barcoded and sequenced utilizing MiSeq (Illumina) Nano kit v.2 as described in [84].

Paired-end sequences were assembled with PandaSeq [85] and processed with the FASTX toolkit. The resulting demultiplexed and collapsed reads were assigned to wells according to barcodes. High-count sequences for every single cell/well were analyzed. Ig heavy chains were aligned to both IMGT [86] and Vbase2 [87] databases, in case of discrepancy IgBLAST was used. $V_{H}$ mutation analyses were restricted to cells with productively rearranged Igh genes, as described in [84]. CDR-H3 analyses were performed as described in [88]. Average hydrophobicity of CDR-H3 was calculated as previously described in [89]. Functional rearrangements were grouped by clonotypes defined by the same $V_{H}$ and $J_{H}$ segment and identical CDR-H3 length and amino acid sequence.

\section{Statistical analyses}

Statistical analyses were performed using GraphPadPrism 7.0 software. Tests were chosen according to the type of variable and indicated in each result. Results with $p>0.05$ were considered significant.

Principal component analysis (PCA) was performed to compare the repertoires globally. Reactivity sections were defined and signal intensities across the sections were quantified and analyzed as described in [90]. 


\section{Acknowledgements}

We thank Dr. Orlando Ferreira for kindly providing ZIKV and DENV EDIII recombinant proteins; Dr. Edgar F.O. de Jesus (in memoriam) and his lab members for cell irradiation and Dr. Garnett Kelsoe for kindly providing the NB21 feeder cells. We thank Dr. Marcelo Bozza for helpful discussion and suggestions. This work was supported by the Brazilian research funding agencies Fundação de Amparo à Pesquisa do Estado do Rio de Janeiro (FAPERJ), Conselho Nacional de Desenvolvimento Científico e Tecnológico (CNPq), Coordenação de Aperfeiçoamento de Pessoal de Nível Superior (CAPES). CBC was supported by CNPq (PhD fellowship), and CAPES (PDSE 88881.132337/2016-01 and Projeto de pesquisa 1759/2014 - Biocomputacional - process number 23038.004628/2014-66). G.D.V. is a BurroughsWellcome Investigator in the Pathogenesis of Infectious Disease.

\section{References}

1. Hunziker, L., et al., Hypergammaglobulinemia and autoantibody induction mechanisms in viral infections. Nat Immunol, 2003. 4(4): p. 343-9.

2. Root-Bernstein, R. and D. Fairweather, Complexities in the relationship between infection and autoimmunity. Curr Allergy Asthma Rep, 2014. 14(1): p. 407.

3. Balakrishnan, T., et al., Dengue virus activates polyreactive, natural lgG $B$ cells after primary and secondary infection. PLoS One, 2011. 6(12): p. e29430.

4. Barbi, L., et al., Prevalence of Guillain-Barré syndrome among Zika virus infected cases: a systematic review and meta-analysis. Braz J Infect Dis, 2018. 22(2): p. 137141.

5. de Oliveira, W.K., et al., Infection-related microcephaly after the 2015 and 2016 Zika virus outbreaks in Brazil: a surveillance-based analysis. Lancet, 2017. 390(10097): p. 861-870.

6. Lardone, R.D., et al., Anti-GM1 lgG antibodies in Guillain-Barré syndrome: fine specificity is associated with disease severity. J Neurol Neurosurg Psychiatry, 2010. 81(6): p. 629-33.

7. Akey, D.L., et al., Flavivirus NS1 structures reveal surfaces for associations with membranes and the immune system. Science, 2014. 343(6173): p. 881-5.

8. Brown, W.C., et al., Extended surface for membrane association in Zika virus NS1 structure. Nat Struct Mol Biol, 2016. 23(9): p. 865-7.

9. Cox, B.D., R.A. Stanton, and R.F. Schinazi, Predicting Zika virus structural biology: Challenges and opportunities for intervention. Antivir Chem Chemother, 2015. 24(34): p. 118-26.

10. Young, P.R., et al., An antigen capture enzyme-linked immunosorbent assay reveals high levels of the dengue virus protein NS1 in the sera of infected patients. J Clin Microbiol, 2000. 38(3): p. 1053-7.

11. Hilgenfeld, R., Zika virus NS1, a pathogenicity factor with many faces. EMBO J, 2016. 35(24): p. 2631-2633.

12. Puerta-Guardo, H., et al., Flavivirus NS1 Triggers Tissue-Specific Vascular Endothelial Dysfunction Reflecting Disease Tropism. Cell Rep, 2019. 26(6): p. 1598-1613.e8.

13. Freire, M.C.L.C., et al., Mapping Putative B-Cell Zika Virus NS1 Epitopes Provides Molecular Basis for Anti-NS1 Antibody Discrimination between Zika and Dengue Viruses. ACS Omega, 2017. 2(7): p. 3913-3920. 
14. Gao, X., et al., Delayed and highly specific antibody response to nonstructural protein 1 (NS1) revealed during natural human ZIKV infection by NS1-based capture ELISA. BMC Infect Dis, 2018. 18(1): p. 275.

15. Stettler, K., et al., Specificity, cross-reactivity, and function of antibodies elicited by Zika virus infection. Science, 2016. 353(6301): p. 823-6.

16. Gonçalves, A.J., et al., Cooperation between CD4+ T Cells and Humoral Immunity Is Critical for Protection against Dengue Using a DNA Vaccine Based on the NS1 Antigen. PLoS Negl Trop Dis, 2015. 9(12): p. e0004277.

17. Richner, J.M., et al., Vaccine Mediated Protection Against Zika Virus-Induced Congenital Disease. Cell, 2017. 170(2): p. 273-283.e12.

18. Bailey, M.J., et al., Antibodies Elicited by an NS1-Based Vaccine Protect Mice against Zika Virus. mBio, 2019. 10(2).

19. Beatty, P.R., et al., Dengue virus NS1 triggers endothelial permeability and vascular leak that is prevented by NS1 vaccination. Sci Transl Med, 2015. 7(304): p. 304ra141.

20. Chuang, Y.C., et al., Dengue Virus Nonstructural Protein 1-Induced Antibodies CrossReact with Human Plasminogen and Enhance Its Activation. J Immunol, 2016. 196(3): p. 1218-26.

21. Lee, P.X., et al., Relative contribution of nonstructural protein 1 in dengue pathogenesis. J Exp Med, 2020. 217(9).

22. Falconar, A.K., The dengue virus nonstructural-1 protein (NS1) generates antibodies to common epitopes on human blood clotting, integrin/adhesin proteins and binds to human endothelial cells: potential implications in haemorrhagic fever pathogenesis. Arch Virol, 1997. 142(5): p. 897-916.

23. Reyes-Sandoval, A. and J.E. Ludert, The Dual Role of the Antibody Response Against the Flavivirus Non-structural Protein 1 (NS1) in Protection and Immuno-Pathogenesis. Front Immunol, 2019. 10: p. 1651.

24. Zellweger, R.M., T.R. Prestwood, and S. Shresta, Enhanced infection of liver sinusoidal endothelial cells in a mouse model of antibody-induced severe dengue disease. Cell Host Microbe, 2010. 7(2): p. 128-39.

25. Lazear, H.M., et al., A Mouse Model of Zika Virus Pathogenesis. Cell Host Microbe, 2016. 19(5): p. 720-30.

26. Yauch, L.E. and S. Shresta, Mouse models of dengue virus infection and disease. Antiviral Res, 2008. 80(2): p. 87-93.

27. Bardina, S.V., et al., Enhancement of Zika virus pathogenesis by preexisting antiflavivirus immunity. Science, 2017. 356(6334): p. 175-180.

28. Demengeot, J., et al., $B$ lymphocyte sensitivity to IgM receptor ligation is independent of maturation stage and locally determined by macrophage-derived IFN-beta. Int Immunol, 1997. 9(11): p. 1677-85.

29. Kiefer, K., et al., Role of type I interferons in the activation of autoreactive $B$ cells. Immunol Cell Biol, 2012. 90(5): p. 498-504.

30. Fallet, B., et al., Interferon-driven deletion of antiviral $B$ cells at the onset of chronic infection. Sci Immunol, 2016. 1(4).

31. Winkler, C.W., et al., Adaptive Immune Responses to Zika Virus Are Important for Controlling Virus Infection and Preventing Infection in Brain and Testes. J Immunol, 2017. 198(9): p. 3526-3535.

32. Pardy, R.D., et al., Analysis of the T Cell Response to Zika Virus and Identification of a Novel CD8+ T Cell Epitope in Immunocompetent Mice. PLoS Pathog, 2017. 13(2): p. e1006184.

33. Huang, $\mathrm{H}$., et al., CD8(+) T Cell Immune Response in Immunocompetent Mice during Zika Virus Infection. J Virol, 2017. 91(22).

34. Dai, L., et al., Structures of the Zika Virus Envelope Protein and Its Complex with a Flavivirus Broadly Protective Antibody. Cell Host Microbe, 2016. 19(5): p. 696-704.

35. Lucas, C.G.O., et al., Critical role of CD4+ T cells and IFNY signaling in antibodymediated resistance to Zika virus infection. Nature Communications, 2018. 9(1): p. 3136. 
36. Hassert, M., et al., CD4+T cells mediate protection against Zika associated severe disease in a mouse model of infection. PLoS Pathog, 2018. 14(9): p. e1007237.

37. Elong Ngono, A., et al., CD4+ $T$ cells promote humoral immunity and viral control during Zika virus infection. PLoS Pathog, 2019. 15(1): p. e1007474.

38. Wardemann, $\mathrm{H}_{\text {., }}$ et al., Predominant autoantibody production by early human $B$ cell precursors. Science, 2003. 301(5638): p. 1374-7.

39. Radic, M.Z. and M. Weigert, Origins of anti-DNA antibodies and their implications for B-cell tolerance. Ann N Y Acad Sci, 1995. 764: p. 384-96.

40. Coelho, S.V.A., et al., Development of standard methods for Zika virus propagation, titration, and purification. J Virol Methods, 2017. 246: p. 65-74.

41. Alvim, R.G.F., I. Itabaiana, and L.R. Castilho, Zika virus-like particles (VLPs): Stable cell lines and continuous perfusion processes as a new potential vaccine manufacturing platform. Vaccine, 2019. 37(47): p. 6970-6977.

42. Beasley, D.W. and A.D. Barrett, Identification of neutralizing epitopes within structural domain III of the West Nile virus envelope protein. J Virol, 2002. 76(24): p. 13097-100.

43. Oliphant, T., et al., Development of a humanized monoclonal antibody with therapeutic potential against West Nile virus. Nat Med, 2005. 11(5): p. 522-30.

44. Shrestha, B., et al., The development of therapeutic antibodies that neutralize homologous and heterologous genotypes of dengue virus type 1. PLoS Pathog, 2010. 6(4): p. e1000823.

45. Watterson, D., N. Modhiran, and P.R. Young, The many faces of the flavivirus NS1 protein offer a multitude of options for inhibitor design. Antiviral Res, 2016. 130: p. 718.

46. Coutelier, J.P., et al., IgG2a restriction of murine antibodies elicited by viral infections. J Exp Med, 1987. 165(1): p. 64-9.

47. Wang, J., et al., A Human Bi-specific Antibody against Zika Virus with High Therapeutic Potential. Cell, 2017. 171(1): p. 229-241.e15.

48. Victora, G.D., et al., Mother-child immunological interactions in early life affect longterm humoral autoreactivity to heat shock protein 60 at age 18 years. J Autoimmun, 2007. 29(1): p. 38-43.

49. Füst, G., et al., Antibodies against heat shock proteins and cholesterol in HIV infection. Mol Immunol, 2005. 42(1): p. 79-85.

50. Quintana, F.J. and I.R. Cohen, The HSP60 immune system network. Trends Immunol, 2011. 32(2): p. 89-95.

51. Nobrega, A., et al., Global analysis of antibody repertoires. II. Evidence for specificity, self-selection and the immunological "homunculus" of antibodies in normal serum. Eur J Immunol, 1993. 23(11): p. 2851-9.

52. Haury, M., et al., Global analysis of antibody repertoires. 1. An immunoblot method for the quantitative screening of a large number of reactivities. Scand J Immunol, 1994. 39(1): p. 79-87.

53. Victora, G.D. and M.C. Nussenzweig, Germinal centers. Annu Rev Immunol, 2012. 30: p. 429-57.

54. Kuraoka, M., et al., Complex Antigens Drive Permissive Clonal Selection in Germinal Centers. Immunity, 2016. 44(3): p. 542-552.

55. Nobrega, A., et al., Functional diversity and clonal frequencies of reactivity in the available antibody repertoire. Eur J Immunol, 1998. 28(4): p. 1204-15.

56. Vale, A.M., et al., $A$ rapid and quantitative method for the evaluation of $V$ gene usage, specificities and the clonal size of B cell repertoires. J Immunol Methods, 2012. 376(12): p. 143-9.

57. Radic, M.Z., et al., B lymphocytes may escape tolerance by revising their antigen receptors. J Exp Med, 1993. 177(4): p. 1165-73.

58. Ivanov, I.I., et al., Development of the expressed Ig CDR-H3 repertoire is marked by focusing of constraints in length, amino acid use, and charge that are first established in early B cell progenitors. J Immunol, 2005. 174(12): p. 7773-80. 
59. Radic, M.Z. and M. Weigert, Genetic and structural evidence for antigen selection of anti-DNA antibodies. Annu Rev Immunol, 1994. 12: p. 487-520.

60. Avirutnan, P., et al., Antagonism of the complement component C4 by flavivirus nonstructural protein NS1. J Exp Med, 2010. 207(4): p. 793-806.

61. Conde, J.N., et al., Inhibition of the Membrane Attack Complex by Dengue Virus NS1 through Interaction with Vitronectin and Terminal Complement Proteins. J Virol, 2016. 90(21): p. 9570-9581.

62. Modhiran, N., et al., A broadly protective antibody that targets the flavivirus NS1 protein. Science, 2021. 371(6525): p. 190-194.

63. Cheng, H.J., et al., Proteomic analysis of endothelial cell autoantigens recognized by anti-dengue virus nonstructural protein 1 antibodies. Exp Biol Med (Maywood), 2009. 234(1): p. 63-73.

64. Akkaya, M., et al., Toll-like receptor 9 antagonizes antibody affinity maturation. Nat Immunol, 2018. 19(3): p. 255-266.

65. Bessa, J., M. Kopf, and M.F. Bachmann, Cutting edge: IL-21 and TLR signaling regulate germinal center responses in a B cell-intrinsic manner. J Immunol, 2010. 184(9): p. 4615-9.

66. Das, A., et al., Follicular Dendritic Cell Activation by TLR Ligands Promotes Autoreactive B Cell Responses. Immunity, 2017. 46(1): p. 106-119.

67. Degn, S.E., et al., Clonal Evolution of Autoreactive Germinal Centers. Cell, 2017. 170(5): p. 913-926.e19.

68. Heesters, B.A., R.C. Myers, and M.C. Carroll, Follicular dendritic cells: dynamic antigen libraries. Nat Rev Immunol, 2014. 14(7): p. 495-504.

69. Sekiguchi, D.R., et al., Development and selection of edited B cells in B6.56R mice. J Immunol, 2006. 176(11): p. 6879-87.

70. Silva-Sanchez, A., et al., Violation of an evolutionarily conserved immunoglobulin diversity gene sequence preference promotes production of dsDNA-specific IgG antibodies. PLoS One, 2015. 10(2): p. e0118171.

71. Muller, D.A. and P.R. Young, The flavivirus NS1 protein: molecular and structural biology, immunology, role in pathogenesis and application as a diagnostic biomarker. Antiviral Res, 2013. 98(2): p. 192-208.

72. Burnett, D.L., et al., Germinal center antibody mutation trajectories are determined by rapid self/foreign discrimination. Science, 2018. 360(6385): p. 223-226.

73. Tas, J.M., et al., Visualizing antibody affinity maturation in germinal centers. Science, 2016. 351(6277): p. 1048-54.

74. Viant, C., et al., Antibody Affinity Shapes the Choice between Memory and Germinal Center B Cell Fates. Cell, 2020. 183(5): p. 1298-1311.e11.

75. Andreano, E. and R. Rappuoli, Immunodominant antibody germlines in COVID-19. J Exp Med, 2021. 218(5).

76. Bastard, P., et al., Autoantibodies against type I IFNs in patients with life-threatening COVID-19. Science, 2020. 370(6515).

77. Andreano, E., et al., Extremely potent human monoclonal antibodies from COVID-19 convalescent patients. Cell, 2021.

78. Kreer, C., et al., Longitudinal Isolation of Potent Near-Germline SARS-CoV-2Neutralizing Antibodies from COVID-19 Patients. Cell, 2020. 182(4): p. 843-854.e12.

79. Vale, A.M., et al., Genetic control of the $B$ cell response to LPS: opposing effects in peritoneal versus splenic $B$ cell populations. Immunogenetics, 2010. 62(1): p. 41-8.

80. Nojima, T., et al., In-vitro derived germinal centre $B$ cells differentially generate memory $B$ or plasma cells in vivo. Nat Commun, 2011. 2: p. 465.

81. Andersson, J., et al., Clonal growth and maturation to immunoglobulin secretion in vitro of every growth-inducible B lymphocyte. Cell, 1977. 10(1): p. 27-34.

82. Taswell, C., Limiting dilution assays for the determination of immunocompetent cell frequencies. I. Data analysis. J Immunol, 1981. 126(4): p. 1614-9.

83. Tiller, T., C.E. Busse, and H. Wardemann, Cloning and expression of murine Ig genes from single $B$ cells. J Immunol Methods, 2009. 350(1-2): p. 183-93. 
84. Mesin, L., et al., Restricted Clonality and Limited Germinal Center Reentry Characterize Memory B Cell Reactivation by Boosting. Cell, 2020. 180(1): p. 92106.e11.

85. Masella, A.P., et al., PANDAseq: paired-end assembler for illumina sequences. BMC Bioinformatics, 2012. 13: p. 31.

86. Lefranc, M.P., et al., IMGT, the international ImMunoGeneTics information system. Nucleic Acids Res, 2009. 37(Database issue): p. D1006-12.

87. Retter, I., et al., VBASE2, an integrative $V$ gene database. Nucleic Acids Res, 2005. 33(Database issue): p. D671-4.

88. Ivanov, II, et al., Development of the expressed lg CDR-H3 repertoire is marked by focusing of constraints in length, amino acid use, and charge that are first established in early B cell progenitors. J Immunol, 2005. 174(12): p. 7773-80.

89. Kyte, J. and R.F. Doolittle, A simple method for displaying the hydropathic character of a protein. J Mol Biol, 1982. 157(1): p. 105-32.

90. Mouthon, L., et al., Invariance and restriction toward a limited set of self-antigens characterize neonatal IgM antibody repertoires and prevail in autoreactive repertoires of healthy adults. Proc Natl Acad Sci U S A, 1995. 92(9): p. 3839-43. 


\section{Figures and Legends}

Figure 1

A

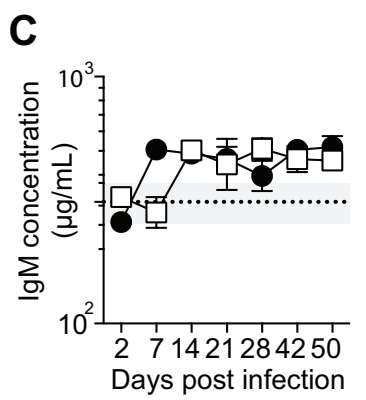

G IgG anti-EDIII ZIKV

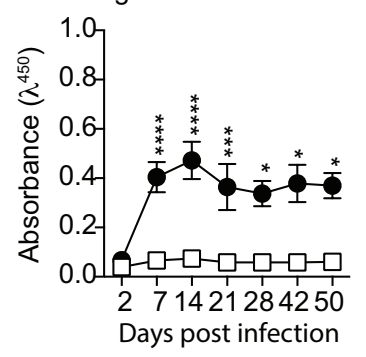

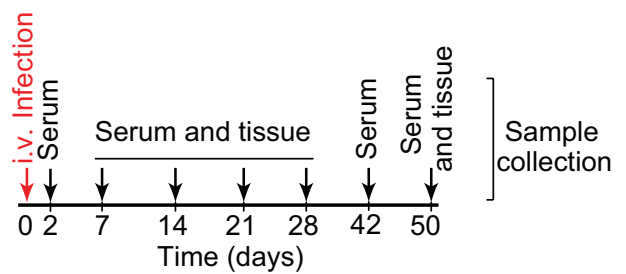

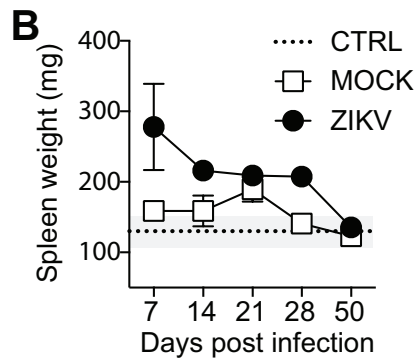

D

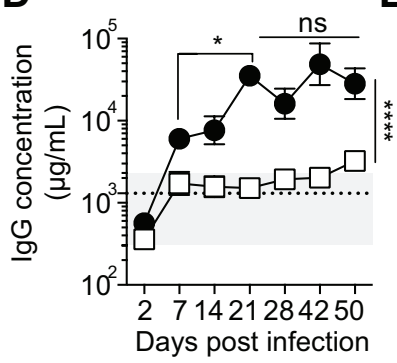

E IgM anti-VLP ZIKV F
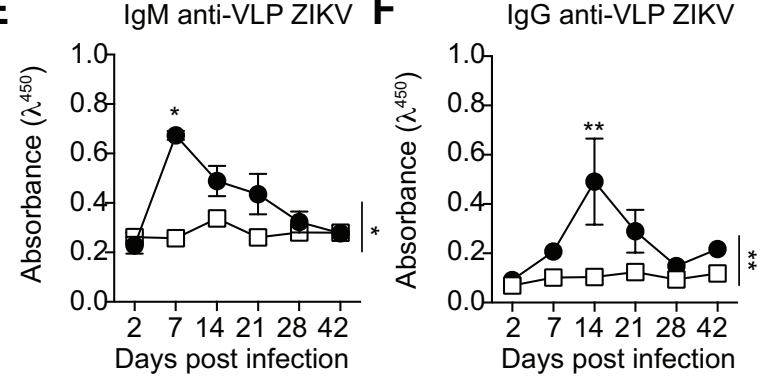

H IgM anti-NS1

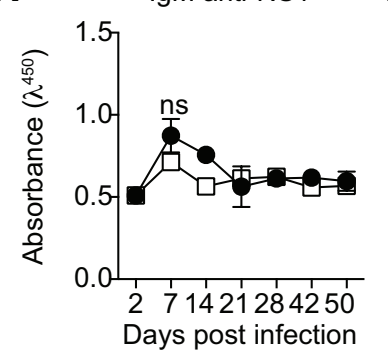

Figure 1. Characterization of ZIKV infection in immunocompetent BALB/c mice. (A) Experimental design indicating the time points of serum samples and lymphoid tissue collections. (B) Spleen weight measured at the time of collection, as indicated. Total serum $\operatorname{lgM}(C)$ and IgG (D) from infected (ZIKV) and control (MOCK) mice, measured by ELISA. (EG) Levels of IgM and IgG specific for viral surface antigens were measured by ELISA (1:120 dilution) utilizing VLPs and recombinant domain III of ZIKV envelope protein (EDIII). (H-I) Levels of IgM (H) and IgG (I) specific for NS1 protein were measured by ELISA (1:120 dilution) utilizing recombinant ZIKV NS1. Data representative of three experiments with eight to sixteen mice per group. ns, not significant; ${ }^{*} p<0,05 ;{ }^{* *} p<0,01 ;{ }^{* * *} p<0,001 ;{ }^{* * * *} p<0,0001$. 


\section{Figure 2}

A $\lg$ anti-NS1

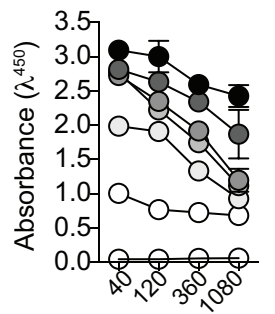

$\ominus 2$ d.p.i.

- 7 d.p.i.

- 14 d.p.i.

- -21 d.p.i.

-o- 28 d.p.i.

- 42 d.p.i.

- 50 d.p.i.

Serum Dilution ${ }^{-1}$

C

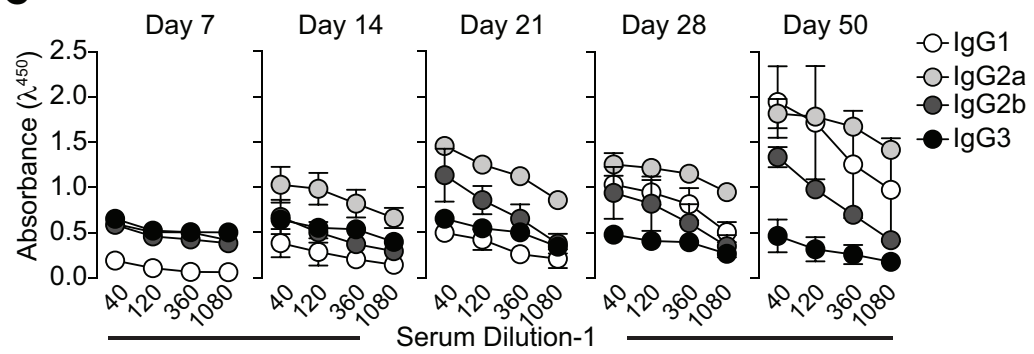

E
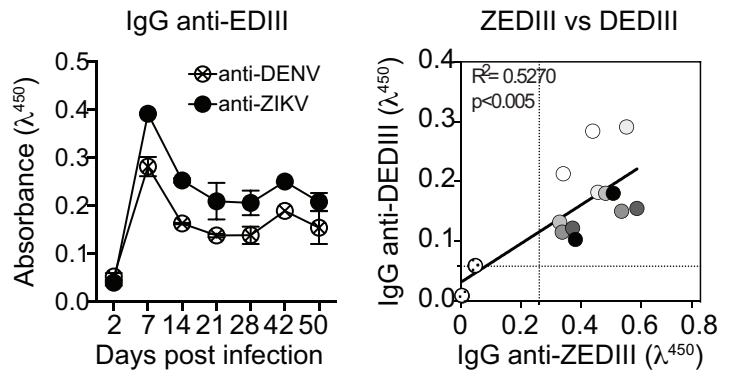

$\mathbf{F}$

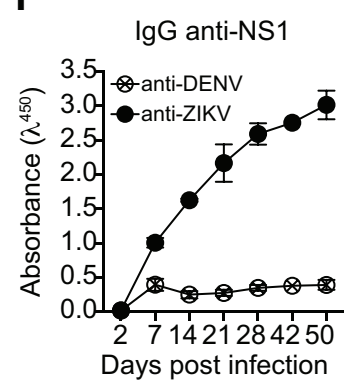

G Serum IgG against muscle extract $\frac{14}{\operatorname{PBSM~Z~}} \frac{21}{\mathrm{MM} \mathrm{Z} \mathrm{Z}} \frac{28}{\mathrm{M} \mathrm{M} \mathrm{Z} \mathrm{Z}} \frac{42}{\mathrm{M} \mathrm{Z} \mathrm{Z}} \frac{50 \text { (d.p.i.) }}{\mathrm{M} \mathrm{Z} \mathrm{Z}}$

\section{H}
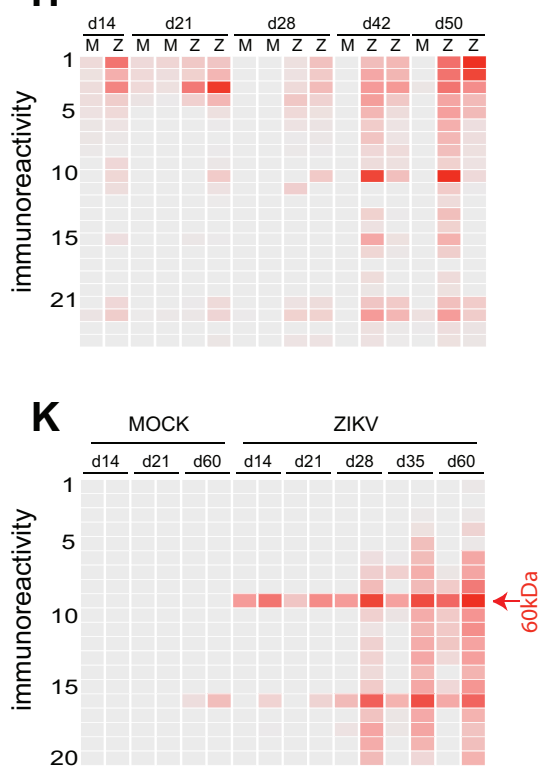

D . O.Total IgG --lsotype Sum

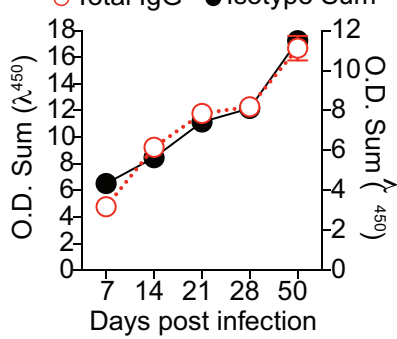

ZNS1 vs DNS1

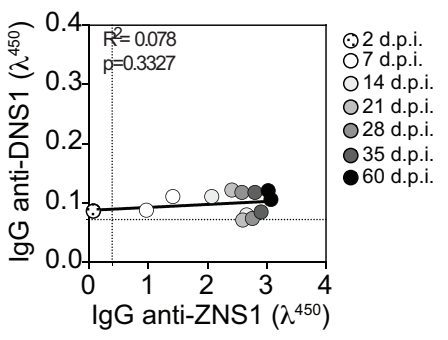

I Average immunoreactivity

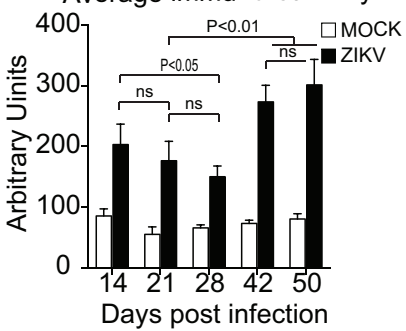

$\mathbf{L}$

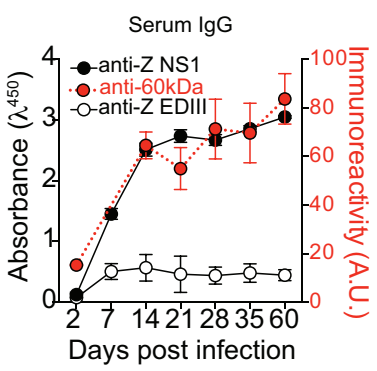

Figure 2. Humoral immune response to NS1 during ZIKV infection correlates with autoreactive antibodies. (A) Binding of serum IgG to ZIKV NS1 protein during infection detected by ELISA. (B) Endpoint titer of serum IgG specific to ZIKV NS1 protein during infection. (C) NS1-specific serum IgG isotypes composition during experimental infection were 
detected by antigen-specific ELISA. (D) Total NS1 specific IgG in serum corresponds to the sum of IgG isotypes, present in distinct proportions after infection. (E-F) Sera from ZIKV infected mice (1:120 dilution) were tested by ELISA for binding to ZIKV and DENV antigens EDIII (E) and NS1 (F). Correlation coefficients show cross-reactivity of EDIII-specific IgG but not of NS1-specific IgG. (G) Self-reactivities present in serum IgG (diluted 1:100) from control $(M)$ and infected $(Z)$ mice using muscle extract from Balb/c mice as source of self-antigens. (H) Intensity of bands was quantified and plotted as a heat map. (I) Total immunoreactivity (sum of all bands intensities) present in the sera of infected (ZIKV) and control (MOCK) mice for each time point after infection. (J) Principal component analysis (PCA) of all self-reactivities at all time points. Red circle indicates the segregation of the control group. (K) Intensity of reactivities present in serum IgG (diluted 1:100) from control (MOCK) and infected mice (ZIKV) using HEp-2 cell extract as source of self-antigens. (L) Intensity of the reactivity to a selected $60 \mathrm{kDa}$ self-antigen throughout time after infection correlates with levels of serum IgG specific to ZIKV NS1 protein but not with levels of IgG specific to domain III of ZIKV envelope protein (ZEDIII). Data for $A$ and $B$ are from one experiment with three mice per group. Data for $C$ and $D$ are from one experiment representative of two experiments with five to sixteen mice per group. Data for $E, F, K$ and $L$ are from one experiment representative of two experiments with two to three mice per group. Data for G-J are from one experiment representative of two experiments with two representative mice per group. ns, not significant. ns, not significant. 


\section{Figure 3}
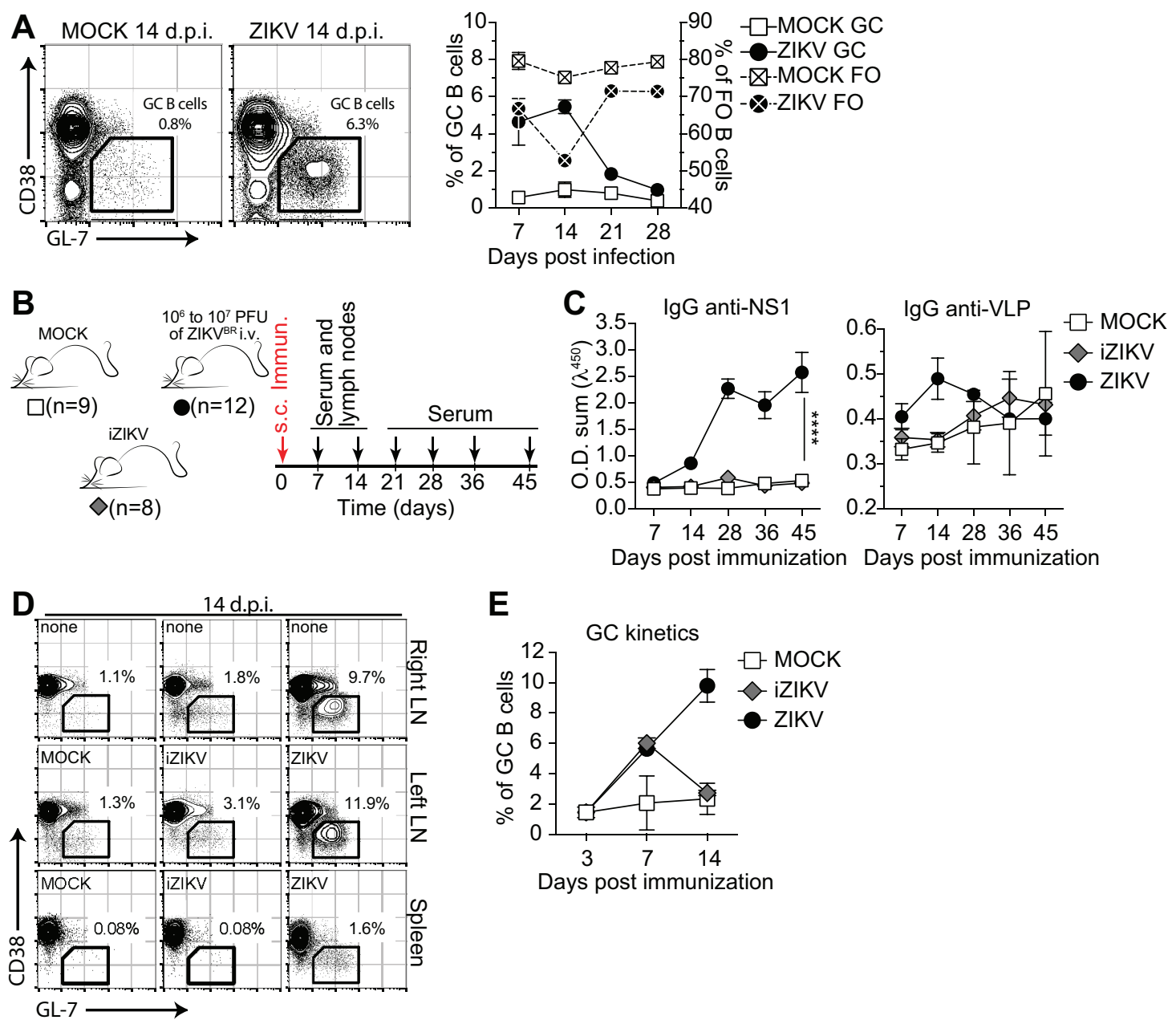

\section{E}

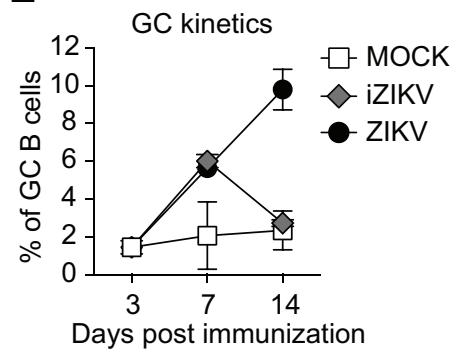

$\mathbf{F}$
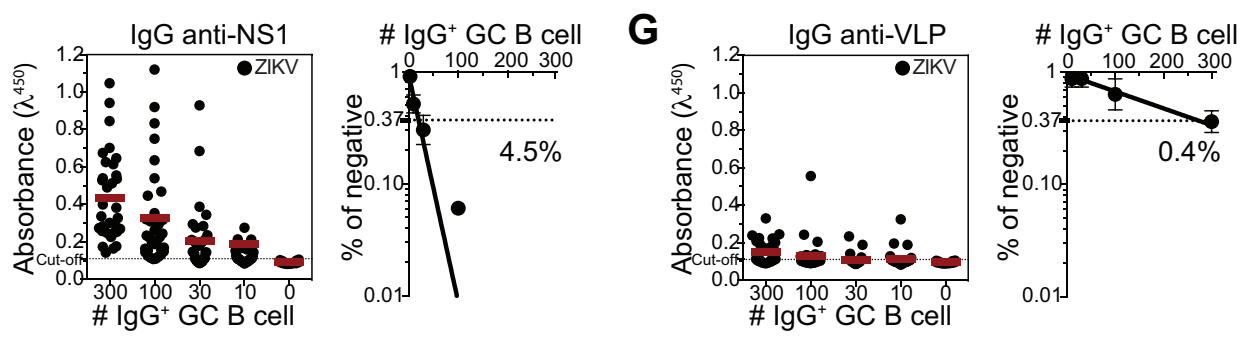

H
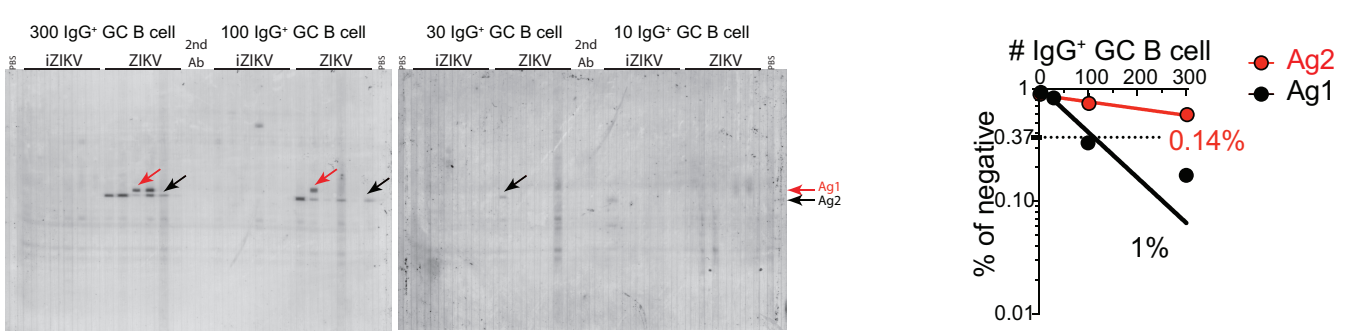

Figure 3. GC B cells produce both virus-specific and autoreactive antibodies. (A) GC B cells (CD38 ${ }^{\text {lo/- }}$ GL-7 $7^{+}$gated on B220 ${ }^{+}$CD138-) in the spleen of ZIKV infected mice at 14 days post infection (left). Kinetics of frequency of follicular (FO) and germinal center (GC) B cells after infection is represented on right panel. (B) Subcutaneous infection experimental design indicating the time points of serum samples and lymphoid tissue collections from control mice 
(MOCK), mice immunized with UV-inactivated virus (iZIKV) and infected mice (ZIKV). (C) Kinetics of serum IgG specific to ZIKV NS1 and VLPs. OD Sum is the summation of ODs of four serum dilutions (1:40, 1:120,1:360 and 1:1080). (D) Representative plots of GC B cells $\left(\mathrm{CD} 38^{\mathrm{lo} /-} \mathrm{GL}-7^{+}\right.$gated on $\left.\mathrm{B}^{2} 20^{+} \mathrm{CD} 138^{-}\right)$at day 14 post infection. Mice were injected in the left footpad. (E) Kinetics of frequency of germinal center (GC) B cells in left popliteal lymph nodes after infection (ZIKV) or immunization (iZIKV). (F-H) GC B cells from popliteal lymph nodes of infected mice were sorted, pooled and cultured in decreasing numbers/well $(300,100,30,10$ cells/well). Supernatants were collected on day 7 and screened for IgG secretion by ELISA. Supernatants that revealed the presence of IgG were tested for antigen specificity by ELISA (F and $\mathbf{G}$ ) or immunoblot against mouse brain tissue as source of self-antigens $(\mathbf{H})$. Frequencies of $\operatorname{lgG}^{+} \mathrm{GC} B$ cells that bound NS1 (F), VLP (G) or self-antigens $(\mathbf{H})$ were calculated utilizing Poisson distribution. Self-antigen reactivities used for frequency determination are indicated by arrows. Cell culture was performed on a monolayer of gammairradiated (20 Gy) NB40L feeder cells (3 x 10 $0^{3}$ cells/well), LPS $(30 \mu \mathrm{g} / \mathrm{mL})$ and IL-21 $(2 \mathrm{ng} / \mathrm{mL})$. Data for $A$ are from one experiment representative of two experiments with eight to sixteen mice per group. Data for D-E are from one experiment representative of two experiments with six to nine mice per group. Data for Fand $G$ are from one experiment representative of two with three mice per group. Data for $\mathrm{H}$ are from a single experiment with three mice per group pooled for each sample. 


\section{Figure 4}
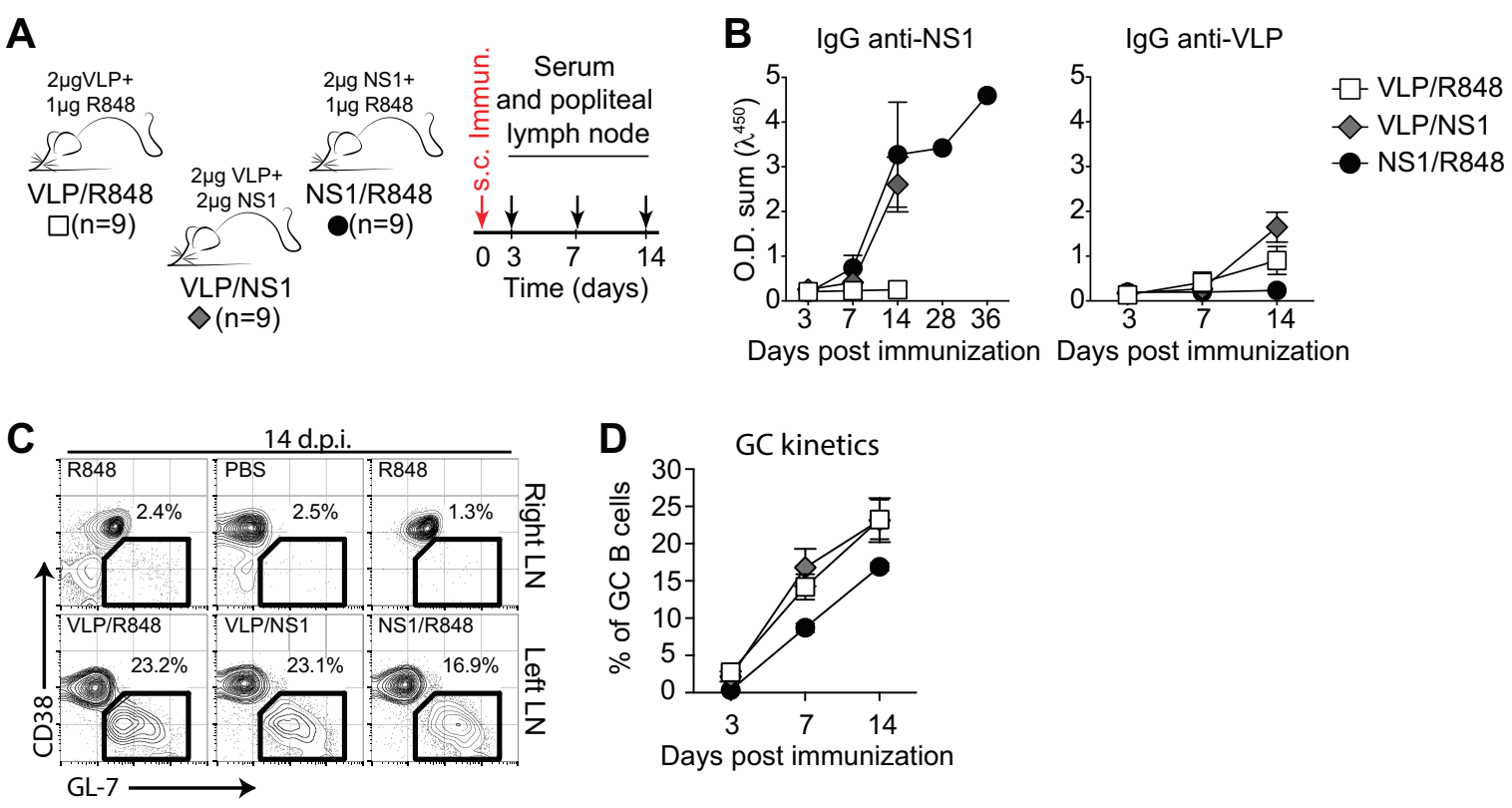

$\mathbf{E}$
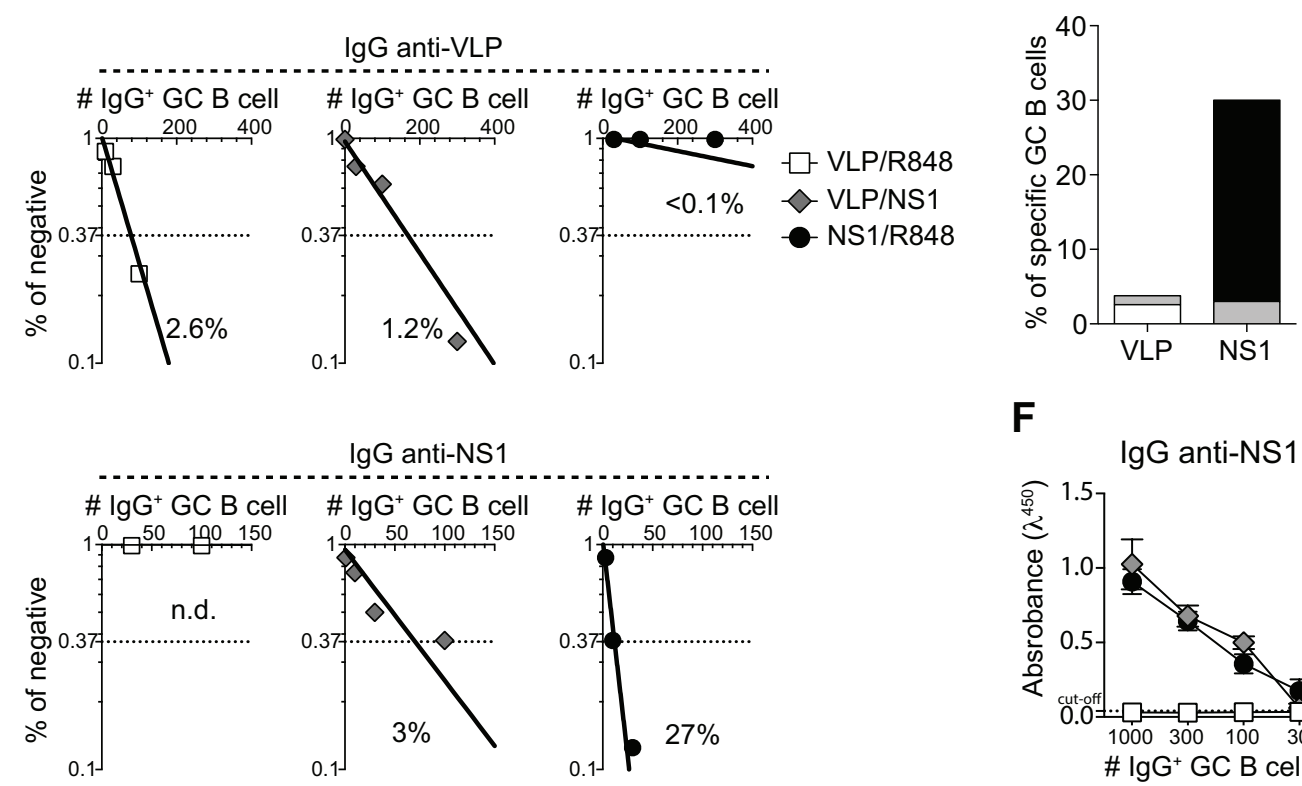

$\lg \mathrm{g}$ anti-NS1

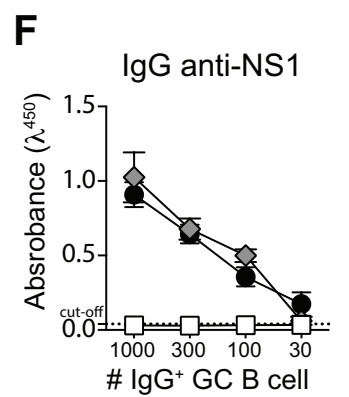

Figure 4. Antigen-specificity of B cells in germinal centers after immunization with ZIKV

VLP and NS1. (A) Experimental design indicating the time points of serum samples and popliteal lymph nodes collections after immunization with NS1 (2 ug/mouse), VLP (2 ug/mouse) or both (2 ug of NS1 and 2 ug of VLP/mouse). Immunizations were adjuvanted with R848 (1ug/mouse). (B) Kinetics of serum levels of IgG binding to ZIKV NS1 recombinant protein or ZIKV VLP, measured by ELISA. OD Sum is the sum of ODs of four serum dilutions $\left(1: 40,1: 120,1: 360\right.$ and 1:1080). (C) Representative plots of GC B cells (CD38 $8^{\text {lo/ }}$ GL- $7^{+}$gated on $\mathrm{B}_{220^{+}} \mathrm{CD}^{\left.138^{-}\right)}$at day 14 post immunization. Mice were immunized in the left footpad. (D) Kinetics of frequency of germinal center (GC) B cells in left popliteal lymph nodes after immunization. (E) GC B cells from popliteal lymph nodes of immunized mice were sorted and cultured in decreasing numbers/well $(300,100,30,10$ cells/well). Supernatants were collected 
on day 7 and screened for IgG secretion by ELISA. Supernatants that revealed the presence of $\lg G$ were tested for antigen specificity by ELISA. Frequencies of $\operatorname{lgG}^{+} \mathrm{GC}$ B cells that bound VLP (upper panel) or NS1 (lower panel) were calculated utilizing Poisson distribution and are summarized on the right graph. Cell culture was performed on a monolayer of gammairradiated (20 Gy) NB40L feeder cells ( $3 \times 10^{3}$ cells/well), LPS $(30 \mu \mathrm{g} / \mathrm{mL})$ and IL-21 $(2 \mathrm{ng} / \mathrm{mL})$. (F) OD of $\mathrm{IgG}^{+}$supernatants of different cell numbers/well binding to ZIKV NS1, measured by ELISA. Data representative of at least two independent experiments with nine mice per group. 


\section{Figure 5}
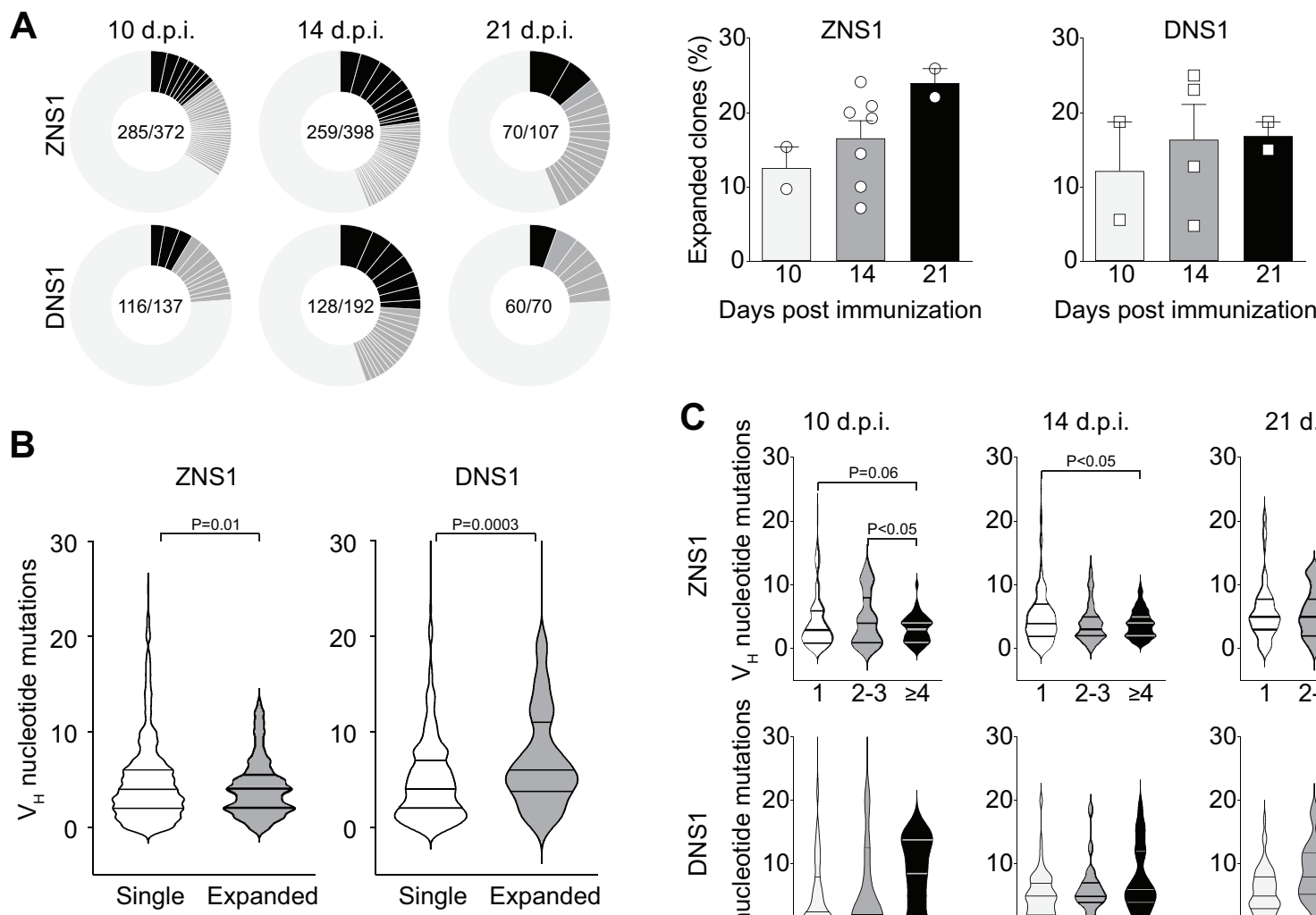

Days post immunization

Days post immunization
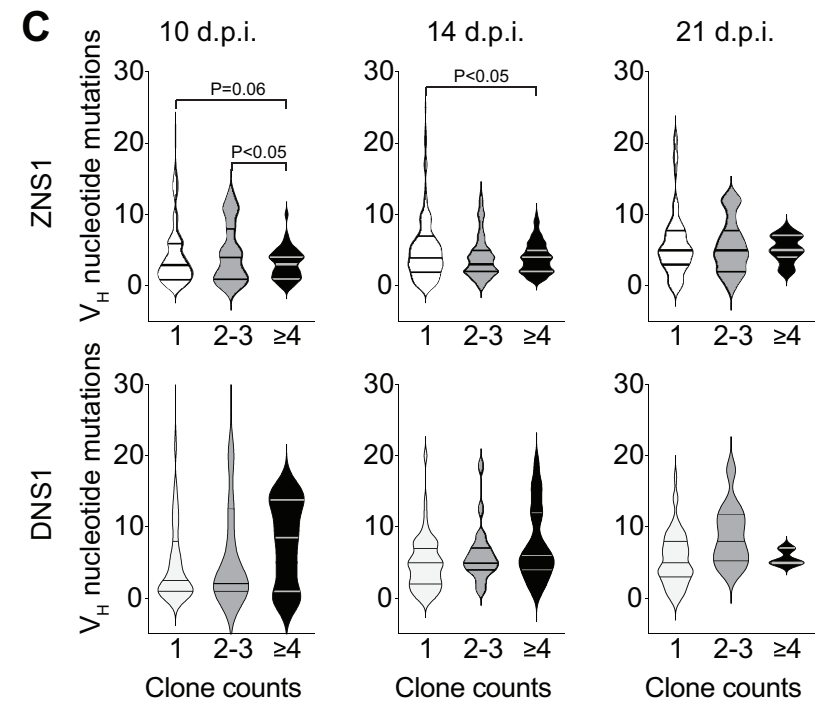

D

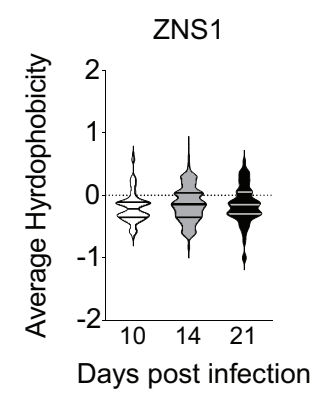

DNS1

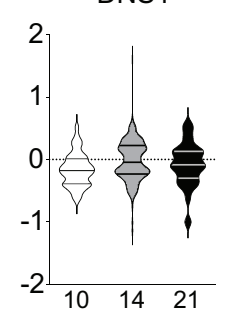

Days post infection
E
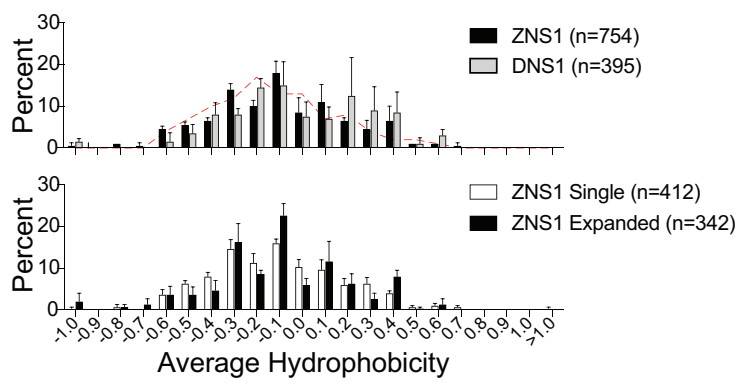
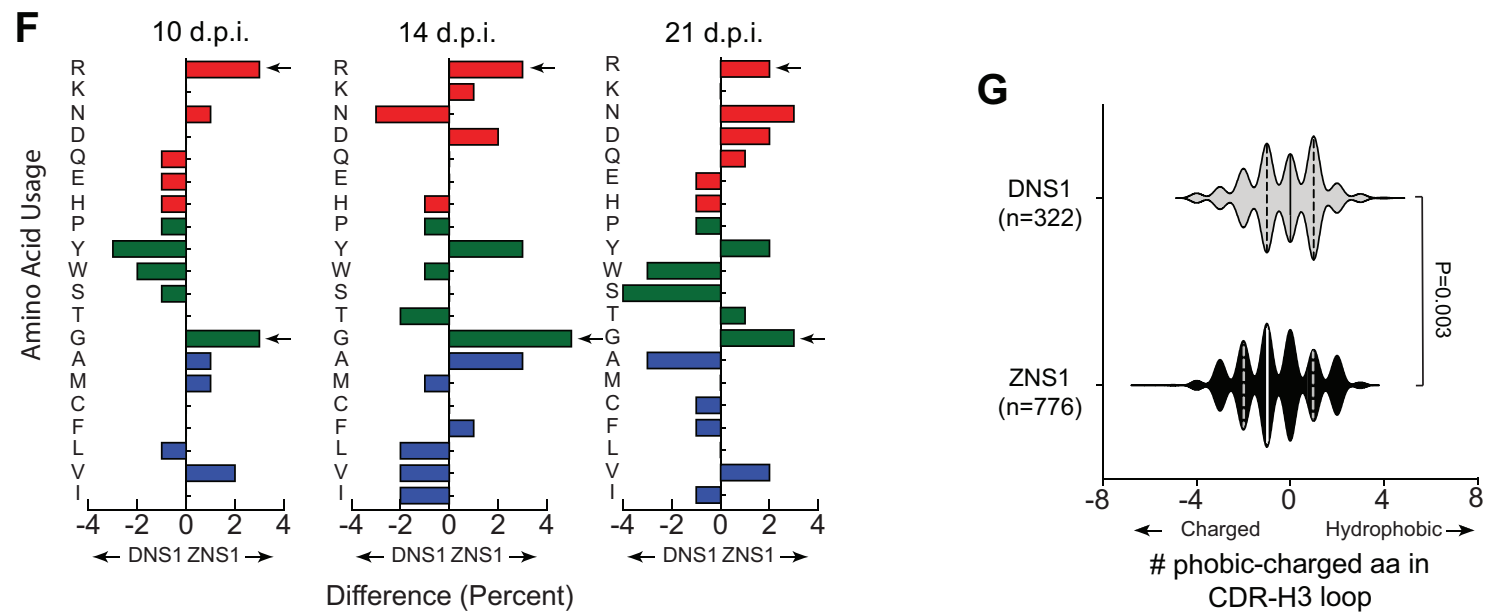

Figure 5. Characterization of $B$ cell repertoire present in GCs after ZIKV NS1 immunization. (A) Single GC B cells from mice immunized s.c. with recombinant ZIKV NS1 
or DENV NS1 were sorted at different time points after immunization and Igh gene was sequenced. Pie charts represent clonal diversity found in all lymph nodes analyzed. Slices represent clonotypes assigned based on $\mathrm{V}_{\mathrm{H}}$ and $\mathrm{J}_{\mathrm{H}}$ usage and $\mathrm{CDRH} 3$ length and sequence. Slice size is proportional to the frequency of each clone. Black slices indicate clone counts higher than 4. Dark gray slices indicate clone counts of 2-3. Light gray where slices are not delimited represents single clones. Proportion of expanded clones is indicated on the right. (B) Number of somatic mutations found in $V_{H}$ segments separated by singletons vs expanded clones. (C) Number of somatic mutations found in $V_{H}$ segments at different time points separated by clone count. (D) $\mathrm{CDR}-\mathrm{H} 3$ average hydrophobicity index variation among all sequences at different time points after immunization. (E) Comparison of CDR-H3 average hydrophobicity index distribution among all sequences from mice immunized with ZIKV NS1 or DENV NS1 (upper panel) and comparison between expanded (clonotypes found more than once in the same lymph node) and single clones from mice immunized with ZIKV NS1 (lower panel). Dashed red line indicates the distribution of $\mathrm{CDR}-\mathrm{H} 3$ average hydrophobicity in follicular B cells from WT BALB/c mice. The normalized Kyte-Doolittle hydrophobicity scale [89] was used to calculate average hydrophobicity. (F) Divergence in the distribution of individual amino acid use in the CDR-H3 loop between ZNS1 versus DNS1 immunized mice at different time points. Red bars indicate charged amino acids, green bars represent neutral amino acids and blue bars, hydrophobic amino acids. (G) Difference in number of hydrophobic and charged amino acids was calculated for each CDR-H3 sequence and distribution was plotted for all sequences from DENV NS1 immunized mice (gray) and ZIKV NS1 immunized mice (black). Lymph nodes were sorted and sequenced individually from 2-5 mice per group at each time point. Sequences were pooled for analyses. 


\section{Figure 6}
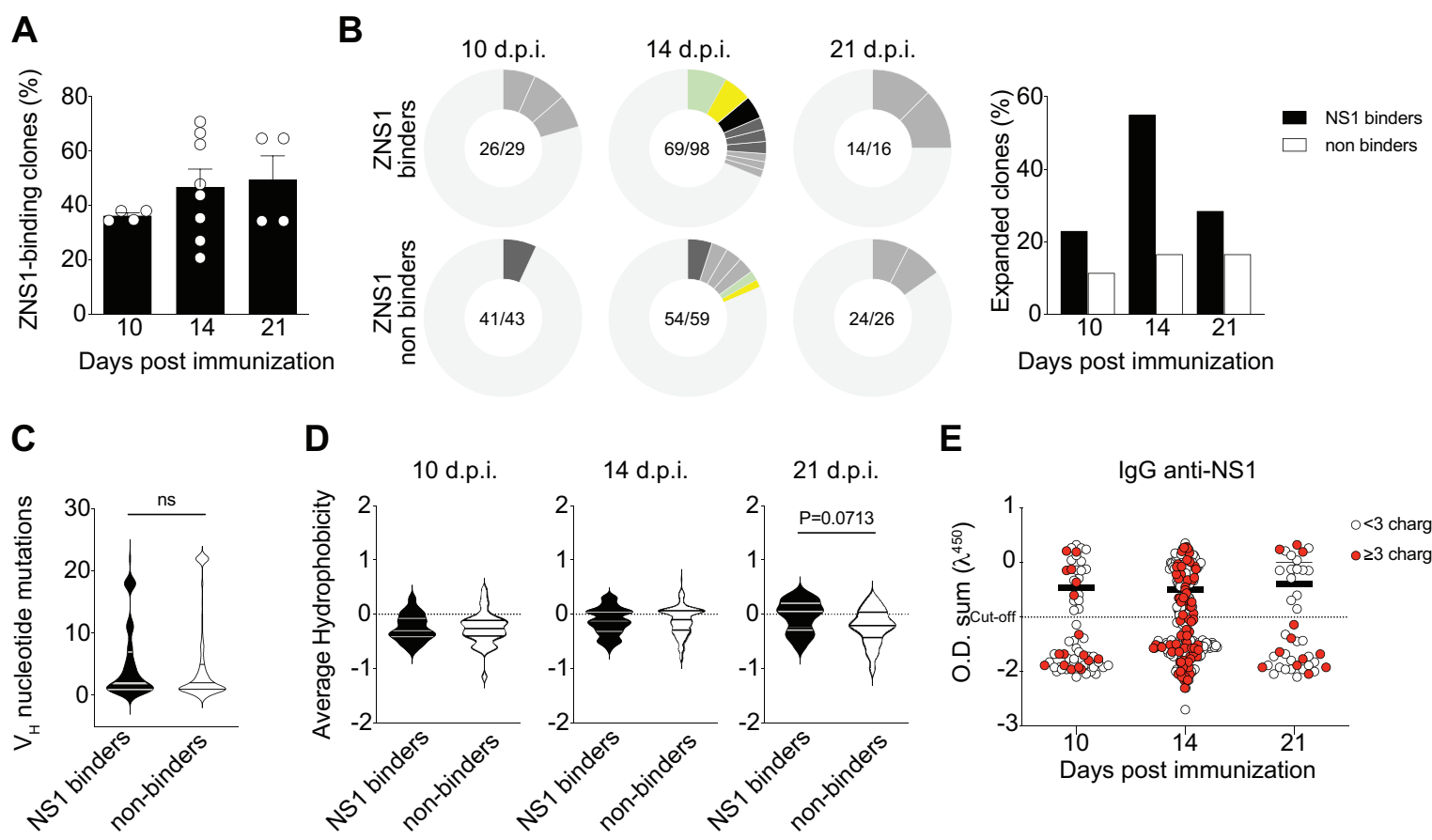

D

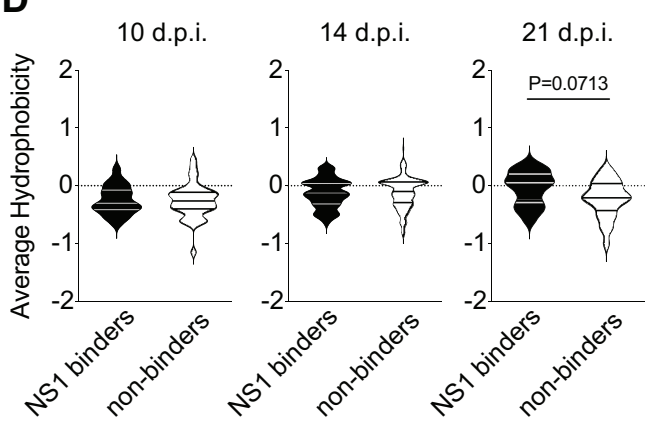

E

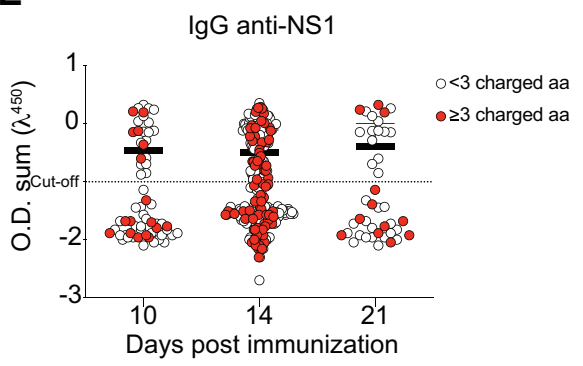

$\mathbf{F}$
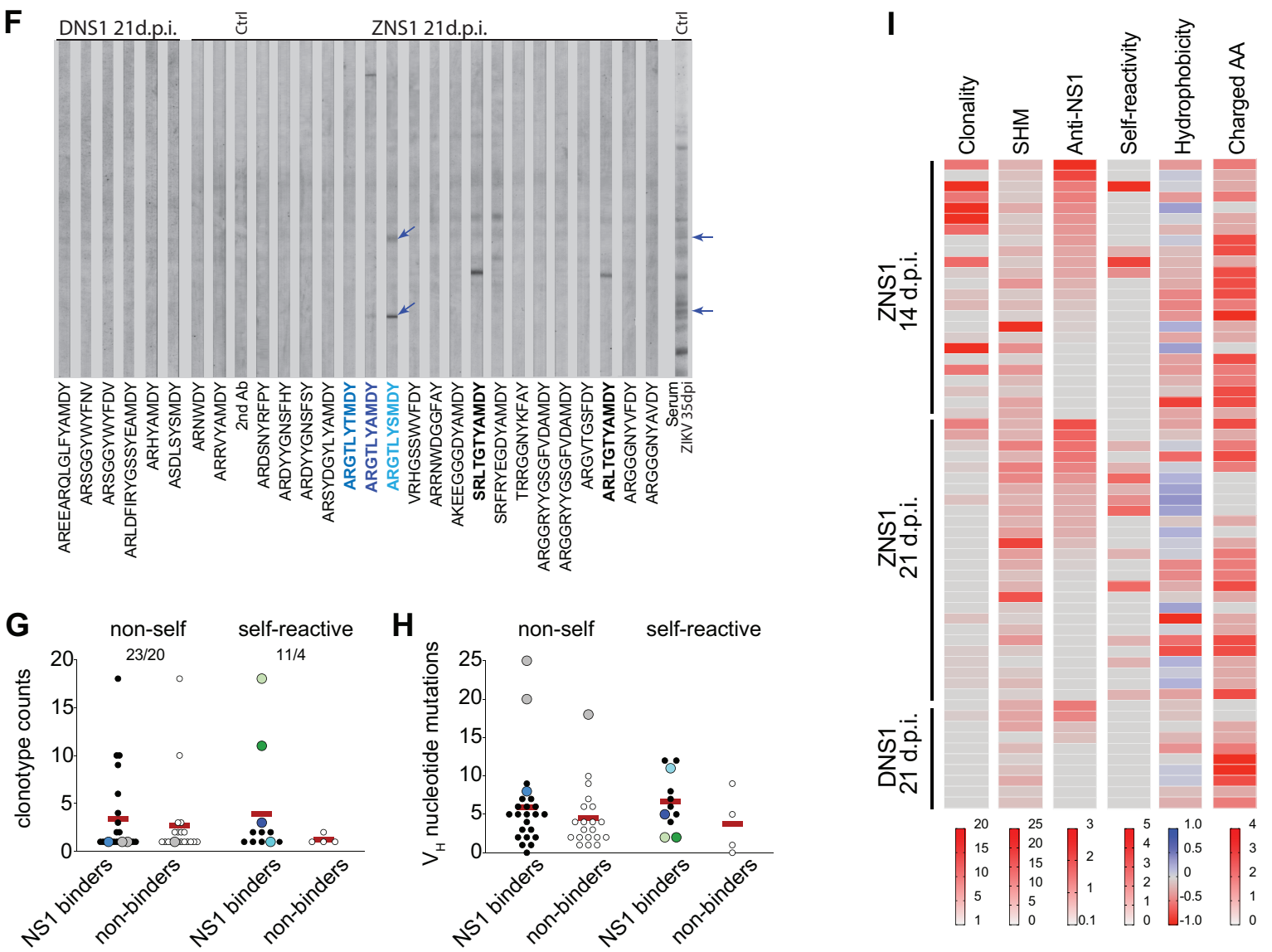

Figure 6. Self-reactivity of GC B cells after ZIKV NS1 immunization. Single GC B cells were sorted and cultured on a monolayer of gamma-irradiated feeder cells ( $1 \times 10^{3}$ cells/well) expressing CD40L, BAFF and IL-21 (Kuraoka et al., 2016). After 7 days, supernatants were collected for binding assays and cells were harvested for Igh sequencing. (A) Frequency of 
$\operatorname{lgG}^{+}$single GC B cell culture supernatants that bound to ZIKV NS1. Supernatants were screened for IgG production and $\mathrm{IgG}^{+}$wells were tested for binding to ZIKV NS1 protein by ELISA. (B) Clonal distribution of GC B cells found to bind to NS1 (upper panel) or that did not bind to NS1 (lower panel). Size of the slice is proportional to the clone frequency. Colored slices represent variants of clones that were found both as binders and non-binders. Right panel represents the frequency of expanded clones among binders and non-binders at specific time points after immunization. (C) Number of somatic mutations found in $V_{H}$ segments from each GC B cell sequenced grouped based on binding to NS1. (D) CDR-H3 average hydrophobicity index variation at different time points grouped by binding to NS1. (E) NS1 binding by ELISA OD related to the presence of charged amino acids. Red dots indicate the presence of 3 or more charged amino acids in CDR-H3 at different time points. (F-I) Single GC B cell culture supernatants were tested for binding to self-antigens by immunoblot. (F) Representative immunoblot profiles of monoclonal IgG from single GC B cell culture supernatants binding to mouse brain extract. Blue arrows indicate immunoreactivities highlighted in the main text (G) Clonotype counts of NS1 binders (black dots) and NS1 non binders (white dots) separated by self-reactivity. Color coded dots represent variants of clones described in the main text. $(\mathrm{H})$ Number of somatic mutations found in $\mathrm{V}_{\mathrm{H}}$ segments grouped by binding to NS1 and self-reactivity. Color coded dots represent variants of clones described in the main text. (I) Clonality, somatic hypermutation, binding to NS1, self-reactivity, hydrophobicity and charged amino acid usage by time after immunization. Clonality corresponds to the number of variants of each clonotype found in the dataset. SHM is represented by the number of $\mathrm{V}_{H}$ mutations found in each sequence. Anti-ZNS1 indicates the OD obtained by ELISA. Self-reactivity corresponds to the number of bands found for each supernatant in mouse tissue extracts (brain and/or muscle). Hydrophobicity corresponds to the average hydrophobicity of the CDR-H3 loop, hydrophobic and charged CDR-H3 sequences are shown in blue and red, respectively. Charged AA indicates the number of charged amino acids found in CDR-H3 loop. Lymph nodes were sorted and cultured individually from 2-3 mice per group at each time point. Sequences were pooled for analyses. 


\section{Supplemental material}

\section{Figure $\mathbf{S 1}$}

A

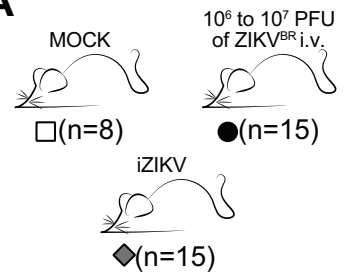

C

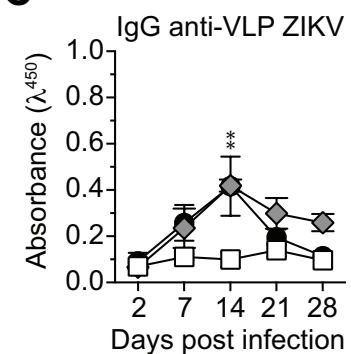

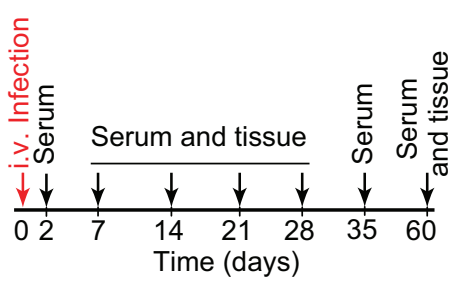

D

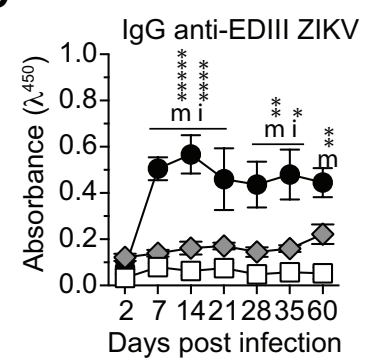

B

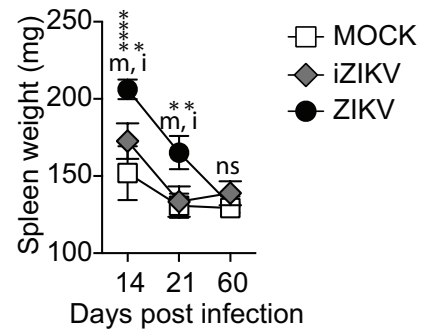

E

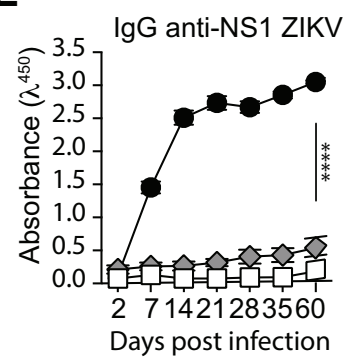

Figure S1. Characterization of humoral immune response to UV-inactivated ZIKV. (A) Experimental design indicating the time points of serum samples and lymphoid tissue collections from control mice (MOCK), mice immunized UV-inactivated virus (iZIKV) and infected mice (ZIKV). (B) Spleen weight measured at the time of collection, as indicated. (CE) Serum levels of IgG specific to VLP (C), domain III of ZIKV envelope protein (D) and NS1 (E). Measured by ELISA at 1:120 dilution. 


\section{Figure S2}

A

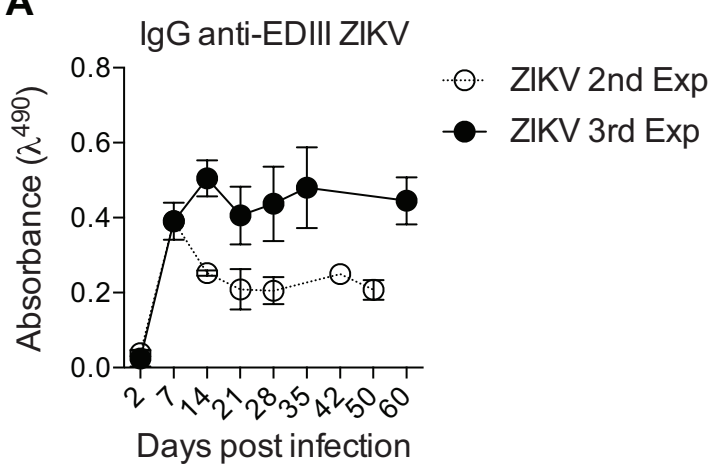

C

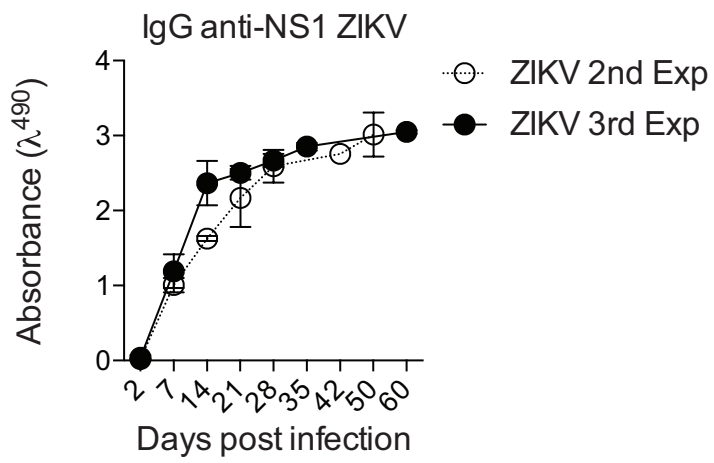

$\mathbf{E}$

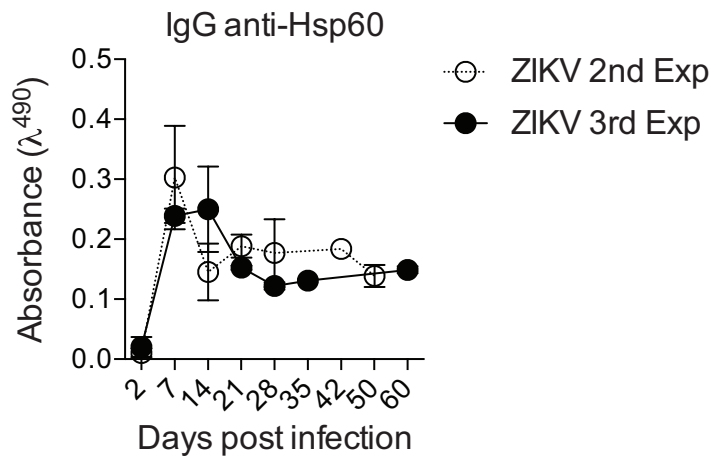

B

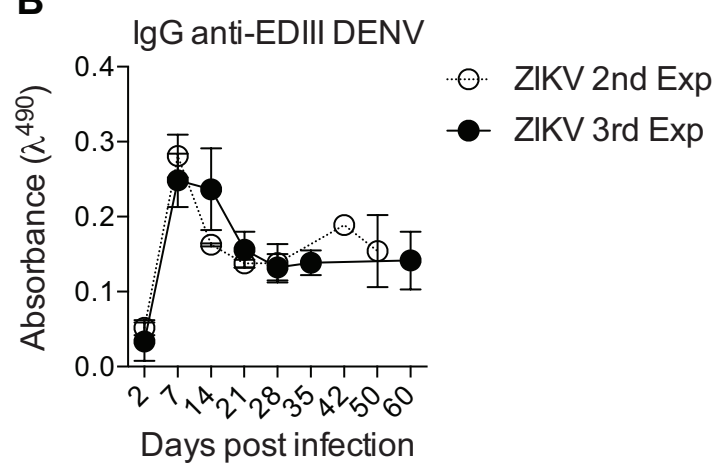

D

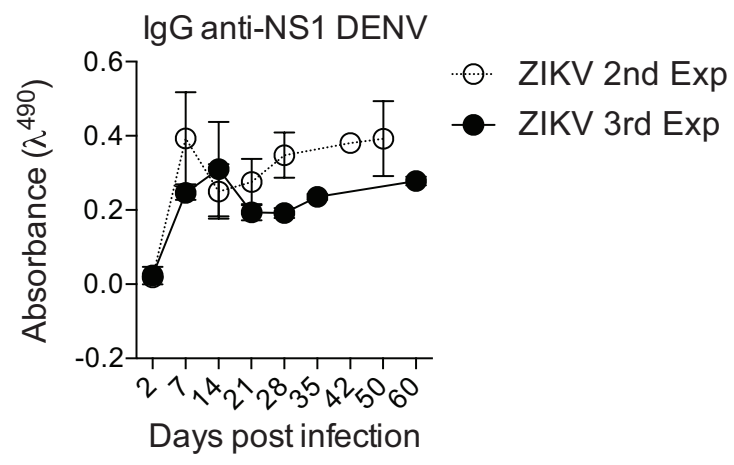

$\mathbf{F}$

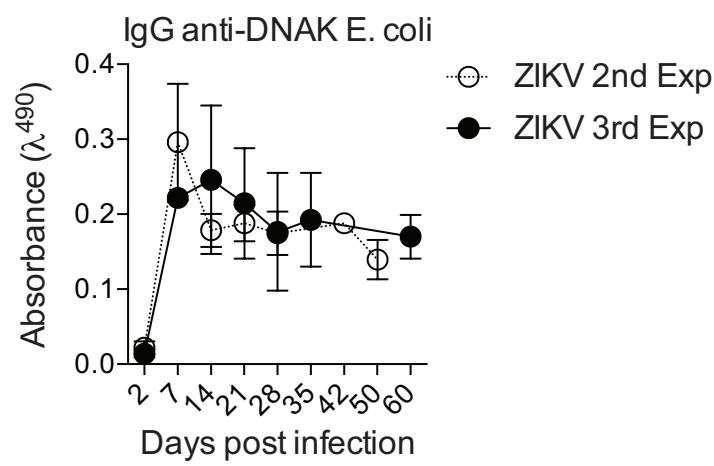

Figure S2. Cross reactivity with unrelated antigens. Sera from ZIKV infected mice (1:120 dilution) from 2 independent experiments were tested by ELISA for binding to ZIKV and DENV antigens EDIII (A and B) and NS1 (C and D) as well as unrelated antigens heat-shock protein Hsp60 and bacterial DNAK from E. coli at different time points. $2^{\text {nd }}$ experiment ended at 50 days and $3^{\text {rd }}$ experiment at 60 days. 


\section{Figure S3}
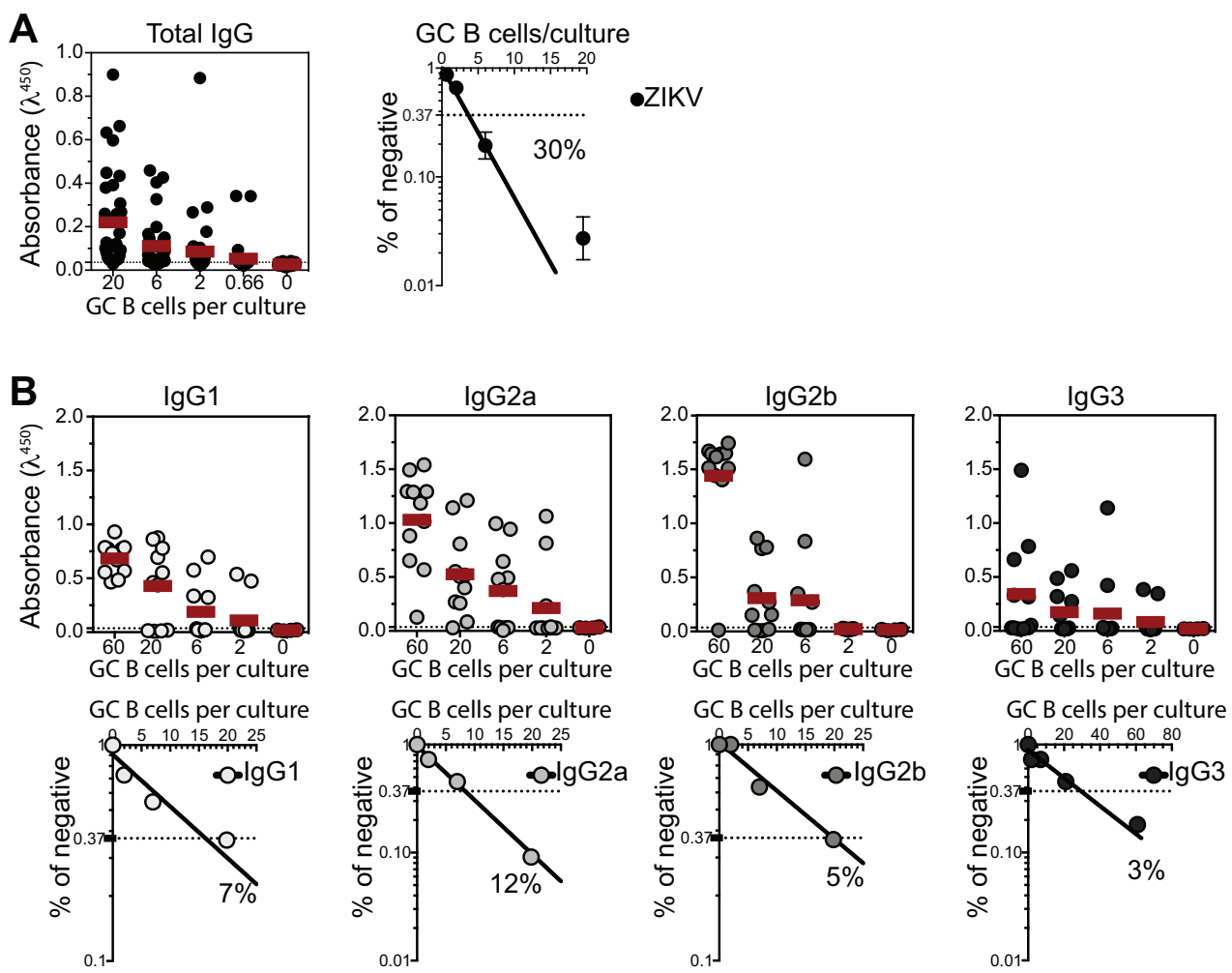

Figure S3. Quantification of the number of responding GC B cell clones per culture. GC $B$ cells from popliteal lymph nodes of ZIKV-infected mice were sorted and cultured in decreasing average number of cells per well $(60,20,6,2,0.66$ cells/well). Supernatants were collected on day 7 and screened for $\lg$ secretion by ELISA. (A) Frequency of GC B cell clones secreting total IgG per culture in response to polyclonal stimuli were calculated using Poisson distribution. (B) Culture supernatants were used to estimate the frequency of GC B cell clones secreting each BALB/c IgG subclasses (IgG1, IgG2a, IgG2b and IgG3). Cell culture was performed on a monolayer of gamma-irradiated (20 Gy) NB40L feeder cells $\left(3 \times 10^{3}\right.$ cells/well), LPS $(30 \mu \mathrm{g} / \mathrm{mL})$ and IL-21 (2 $\mathrm{ng} / \mathrm{mL})$. 


\section{Figure S4}

A

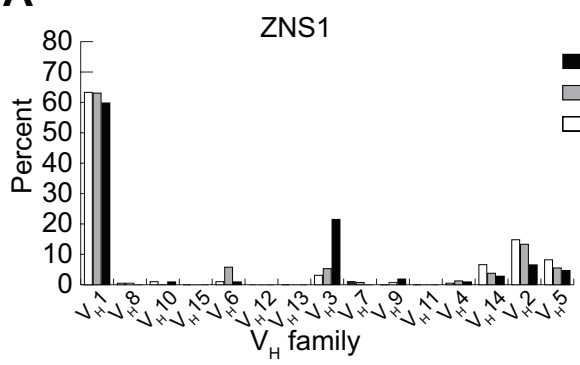

B

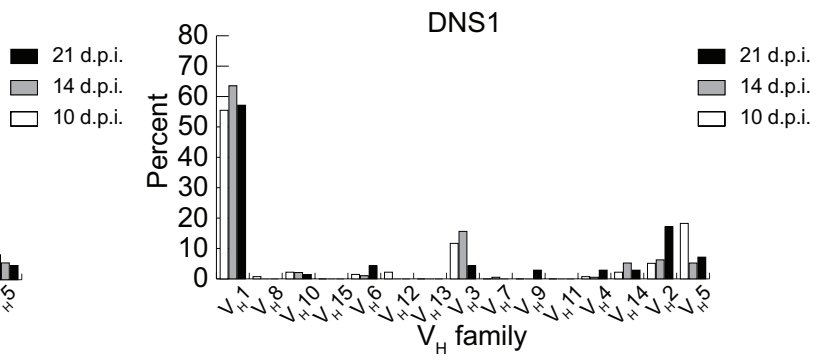

\section{C}

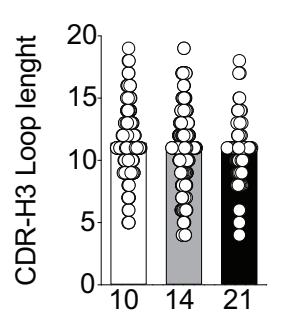

Days post infection

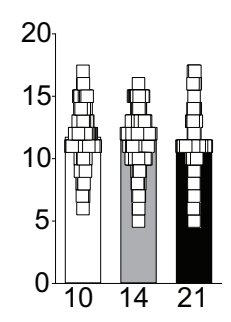

Days post infection

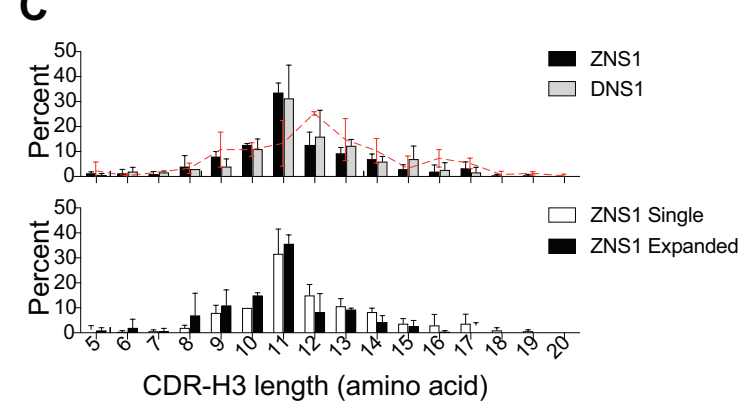

Figure S4. Analysis of $\mathrm{V}_{\mathrm{H}}$ family usage and CDR-H3 length of Igh transcripts from $B$ cells present in GCs after ZIKV NS1 or DENV NS1 immunization. (A) Shown here is the expression of each $V_{H}$ family as a percentage of total functional transcripts from GC B cells at different time points after immunization with ZIKV NS1 or DENV NS1. (B) CDR-H3 loop length variation at different time points after immunization with ZIKV NS1 or DENV NS1. (C) CDRH3 loop length distribution of all sequences at all time points (upper panel) and comparison between clonal (clonotypes found more than once in the same lymph node) and single clones from mice immunized with ZIKV NS1 (lower panel). Dashed red line indicates the distribution of CDR-H3 length in follicular B cells from WT BALB/c mice. 


\section{Figure S5}

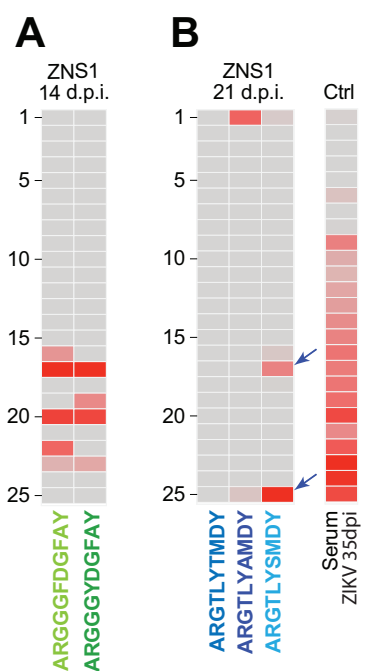

Figure S5. Heatmaps showing the pattern of self-reactivities of two particular clonal families. (A) Immunoreactivity profile of two poly-reactive variants from the clonal family bearing "glycine-enriched CDR-H3" (ARGGGYDGFAY) found among GC B cells from mice 14 d.p.i. with ZIKV NS1. (B) Immunoreactivity profile of the autoreactive clonotype ARGTLYAMDY and its two more mutated variants: ARGTLYTMDY (non-autoreactive) and ARGTLYSMDY (autoreactive). 
bioRxiv preprint doi: https://doi.org/10.1101/2020.12.24.423863; this version posted March 13,2021 . The copyright holder for this preprint

(which was not certified by peer review) is the author/funder, who has granted bioRxiv a license to display the preprint in perpetuity. It is made available under aCC-BY-NC-ND 4.0 International license.

Supplementary Table 1: CDR-H3 characteristics from germinal center B cell clones tested for self-reactivity.

\begin{tabular}{|c|c|c|c|c|c|c|c|c|c|c|c|c|c|}
\hline ID & $\begin{array}{l}\text { Immunization } \\
\text { antigen }\end{array}$ & dpi & $\begin{array}{l}\text { Clone } \\
\text { count }\end{array}$ & $\begin{array}{l}\text { VH } \\
\text { mut \# }\end{array}$ & CDR-H3 aa sequence & VH & JH & $\begin{array}{l}\text { CDR-H3 } \\
\text { loop } \\
\text { length }\end{array}$ & $\begin{array}{c}\text { Average } \\
\text { hidrophobicity }\end{array}$ & $\begin{array}{c}\text { CDR-H3 } \\
\text { charged aa \# }\end{array}$ & $\begin{array}{l}\text { Total IgG } \\
\text { OD }\end{array}$ & $\begin{array}{l}\text { Anti-NS1 } \\
\text { IgG OD }\end{array}$ & $\begin{array}{c}\text { \# of self } \\
\text { reactive } \\
\text { bands }\end{array}$ \\
\hline P07A02 & ZIKV NS1 & 14 & 10 & 5 & ARDRYGAMDY & V2-9 & 4 & 10 & -0.354 & 2 & 2.623 & 2.873 & 0 \\
\hline P05F01 & ZIKV NS1 & 14 & 1 & 3 & VRGAHYAMDY & V1-7 & 4 & 10 & 0.078 & 1 & 2.453 & 2.276 & 0 \\
\hline P05B07 & ZIKV NS1 & 14 & 18 & 2 & ARGGGFDGFAY & V1-12 & 3 & 11 & 0.037 & 1 & 1.971 & 1.491 & 5 \\
\hline P07A05 & ZIKV NS1 & 14 & 10 & 2 & ARDRYGAMDY & V2-9 & 4 & 10 & -0.354 & 2 & 2.723 & 1.462 & 0 \\
\hline $\mathrm{P} 05 \mathrm{CO} 05$ & ZIKV NS1 & 14 & 18 & 6 & ARGGGLWGAMDY & V1-87 & 4 & 12 & 0.307 & 0 & 1.713 & 1.256 & 0 \\
\hline P05F10 & ZIKV NS1 & 14 & 1 & 1 & ARDRATVVADYYTMDY & V2-6-7 & 4 & 16 & 0.069 & 3 & 1.827 & 1.037 & 0 \\
\hline P05G12 & ZIKV NS1 & 14 & 1 & 5 & VRGQIHYYGPWYFDV & V1-87 & 1 & 15 & -0.147 & 3 & 2.764 & 0.859 & 1 \\
\hline $\mathrm{P} 05 \mathrm{CO}$ & ZIKV NS1 & 14 & 11 & 2 & ARGGGYDGFAY & V1-12 & 3 & 11 & -0.192 & 1 & 2.035 & 0.85 & 4 \\
\hline $\mathrm{P} 05 \mathrm{HO3}$ & ZIKV NS1 & 14 & 2 & 4 & ARGQFHYYGPWYFDV & V1-87 & 1 & 15 & -0.207 & 3 & 2.23 & 0.841 & 2 \\
\hline P05D07 & ZIKV NS1 & 14 & 1 & 9 & ARSGGSYDNYDYCSMDY & V1-54 & 4 & 17 & -0.254 & 3 & 2.099 & 0.664 & 0 \\
\hline P05G06 & ZIKV NS1 & 14 & 3 & 2 & ARKKDGYAMDY & V2-6-4 & 4 & 11 & -0.445 & 3 & 2.761 & 0.531 & 0 \\
\hline P07A10 & ZIKV NS1 & 14 & 4 & 4 & ARERYGSSLAY & V1-14 & 3 & 11 & -0.457 & 2 & 2.524 & 0.369 & 0 \\
\hline P05E07 & ZIKV NS1 & 14 & 1 & 1 & ARGKRDGYYPYAMDY & V2-6-7 & 4 & 15 & -0.375 & 4 & 2.35 & 0.228 & 0 \\
\hline P05G04 & ZIKV NS1 & 14 & 1 & 25 & ARMGLDSSGLYGMDY & V1S132 & 4 & 15 & 0.222 & 1 & 2.341 & 0.226 & 0 \\
\hline P05F11 & ZIKV NS1 & 14 & 2 & 2 & AREYYGSTFAY & V1-14 & 3 & 11 & -0.28 & 1 & 2.577 & 0.164 & 0 \\
\hline $\mathrm{P} 05 \mathrm{C} 12$ & ZIKV NS1 & 14 & 18 & 10 & ARGGGLWGAMDY & V1-87 & 4 & 12 & 0.307 & 0 & 1.926 & 0.08 & 0 \\
\hline P07B12 & ZIKV NS1 & 14 & 1 & 1 & ARDDGYSTYHYAMDY & V1-87 & 4 & 15 & -0.309 & 3 & 2.308 & 0.039 & 0 \\
\hline P07B01 & ZIKV NS1 & 14 & 10 & 9 & ARDRYGAMDY & V2-9 & 4 & 10 & -0.354 & 2 & 1.67 & 0.028 & 0 \\
\hline P05E04 & ZIKV NS1 & 14 & 1 & 4 & TRGGGYDYEAWFAY & V5-6-4 & 3 & 14 & -0.202 & 2 & 1.775 & 0.009 & 0 \\
\hline P05G03 & ZIKV NS1 & 14 & 3 & 2 & ARQVRPYAMDY & V3-2 & 4 & 11 & -0.095 & 3 & 2.234 & 0.008 & 0 \\
\hline P05B06 & ZIKV NS1 & 14 & 1 & 7 & AREDRGFAY & V5-6-3 & 3 & 9 & -0.818 & 3 & 1.547 & 0.005 & 0 \\
\hline P05G08 & ZIKV NS1 & 14 & 2 & 6 & AREYYGSTFAY & V1-14 & 3 & 11 & -0.28 & 1 & 2.252 & 0.005 & 0 \\
\hline P09A08 & ZIKV NS1 & 21 & 9 & 5 & AQQRLPYYFDY & V3-1 & 2 & 11 & -0.302 & 3 & 0.818 & 2.131 & 0 \\
\hline P09A04 & ZIKV NS1 & 21 & 6 & 5 & ARNWAFTY & V1-9 & 3 & 8 & -0.123 & 1 & 0.405 & 1.852 & 0 \\
\hline P09F10 & ZIKV NS1 & 21 & 2 & 12 & ARGGRYYGSGFVDAMDY & V1-67 & 4 & 17 & 0.054 & 2 & 0.784 & 1.641 & 1 \\
\hline P09F08 & ZIKV NS1 & 21 & 1 & 5 & TRRGGNYKFAY & V4-1 & 3 & 11 & -0.602 & 3 & 0.492 & 1.581 & 0 \\
\hline P09G02 & ZIKV NS1 & 21 & 2 & 12 & ARGGRYYGSGFVDAMDY & V1-67 & 4 & 17 & 0.054 & 2 & 0.677 & 1.365 & 1 \\
\hline P09B04 & ZIKV NS1 & 21 & 1 & 11 & ARGTLYSMDY & V1-87 & 4 & 10 & 0.198 & 0 & 0.626 & 1.107 & 3 \\
\hline P09G06 & ZIKV NS1 & 21 & 1 & 6 & ARLTGTYAMDY & V1-55 & 4 & 11 & 0.298 & 0 & 0.87 & 0.764 & 1 \\
\hline P09B02 & ZIKV NS1 & 21 & 3 & 5 & ARGTLYAMDY & V1-87 & 4 & 10 & 0.372 & 0 & 0.954 & 0.756 & 2 \\
\hline P09F04 & ZIKV NS1 & 21 & 1 & 7 & SRLTGTYAMDY & V1-55 & 4 & 11 & 0.298 & 0 & 0.564 & 0.732 & 3 \\
\hline P09A07 & ZIKV NS1 & 21 & 1 & 5 & SRGGYDGGAYYFDY & V1-39 & 2 & 14 & -0.102 & 1 & 0.834 & 0.723 & 0 \\
\hline P09B01 & ZIKV NS1 & 21 & 1 & 8 & ARGTLYTMDY & V1-87 & 4 & 10 & 0.204 & 0 & 0.642 & 0.716 & 0 \\
\hline P09B06 & ZIKV NS1 & 21 & 1 & 20 & VRHGSSWVFDY & V1S40 & 2 & 11 & 0.063 & 1 & 0.367 & 0.518 & 0 \\
\hline P09A02 & ZIKV NS1 & 21 & 1 & 8 & ARHGISQAWFAY & V1-54 & 3 & 12 & 0.05 & 2 & 0.854 & 0.253 & 1 \\
\hline P09A10 & ZIKV NS1 & 21 & 1 & 6 & ARDYYGNSFSY & V9-2-1 & 2 & 11 & -0.435 & 2 & 0.578 & 0.204 & 0 \\
\hline P09A09 & ZIKV NS1 & 21 & 1 & 3 & ARDYYGNSFHY & V9-2-1 & 2 & 11 & -0.435 & 2 & 0.529 & 0.142 & 0 \\
\hline P09F05 & ZIKV NS1 & 21 & 1 & 5 & SRFRYEGDYAMDY & V1-82 & 4 & 13 & -0.243 & 3 & 0.905 & 0.041 & 3 \\
\hline P09F01 & ZIKV NS1 & 21 & 1 & 18 & ARGGGNYAVDY & V1-18 & 4 & 11 & -0.068 & 1 & 0.424 & 0.021 & 0 \\
\hline P09G04 & ZIKV NS1 & 21 & 1 & 2 & ARGVTGSFDY & V1-54 & 2 & 10 & 0.298 & 0 & 0.973 & 0.019 & 0 \\
\hline P09A01 & ZIKV NS1 & 21 & 3 & 1 & ARNWDY & V2-2 & 2 & 6 & -1 & 1 & 0.563 & 0.015 & 0 \\
\hline P09A11 & ZIKV NS1 & 21 & 1 & 4 & ARSYDGYLYAMDY & V3-8 & 4 & 13 & 0.036 & 1 & 0.659 & 0.012 & 0 \\
\hline P09E11 & ZIKV NS1 & 21 & 1 & 9 & ARRNWDGGFAY & V5-12-1 & 3 & 11 & -0.563 & 3 & 0.189 & 0.012 & 1 \\
\hline P09G03 & ZIKV NS1 & 21 & 2 & 2 & ARDSNYRFPY & V1-26 & 3 & 10 & -0.734 & 3 & 0.502 & 0.012 & 0 \\
\hline P09E09 & ZIKV NS1 & 21 & 2 & 0 & ARRVYAMDY & V3-2 & 4 & 9 & 0.2 & 1 & 0.837 & 0.011 & 1 \\
\hline P09A05 & ZIKV NS1 & 21 & 2 & 2 & ARGGGNYVFDY & V1S56 & 2 & 11 & 0.07 & 1 & 0.47 & 0.01 & 0 \\
\hline P09E02 & ZIKV NS1 & 21 & 2 & 4 & ARRVYAMDY & V3-2 & 4 & 9 & 0.2 & 1 & 0.775 & 0.009 & 0 \\
\hline P09F02 & ZIKV NS1 & 21 & 1 & 1 & AKEEGGGDYAMDY & V1-7 & 4 & 13 & -0.301 & 3 & 0.893 & 0.009 & 1 \\
\hline P09C07 & DENV NS1 & 21 & 1 & 6 & ARSGGYWYFNV & V1S29 & 1 & 11 & -0.12 & 0 & 0.915 & 1.516 & 0 \\
\hline P09D10 & DENV NS1 & 21 & 2 & 7 & ARSGGYWYFDV & V1S29 & 1 & 11 & -0.12 & 0 & 0.674 & 1.319 & 0 \\
\hline $\mathrm{P} 09 \mathrm{HO}$ & DENV NS1 & 21 & 1 & 7 & ASDLSYSMDY & V2-9 & 4 & 10 & -0.014 & 1 & 0.695 & 0.474 & 0 \\
\hline P09H11 & DENV NS1 & 21 & 1 & 0 & ARRWAYYYGSSGYYFDY & V5-12-1 & 2 & 17 & -0.18 & 1 & 0.324 & 0.438 & 0 \\
\hline P09HO6 & DENV NS1 & 21 & 1 & 4 & AREGYGNSFDY & V1-14 & 3 & 11 & -0.385 & 2 & 0.934 & 0.027 & 0 \\
\hline P09C01 & DENV NS1 & 21 & 1 & 1 & ARLDFIRYGSRYEAMDY & V5-9-2 & 4 & 17 & -0.02 & 4 & 0.919 & 0.014 & 0 \\
\hline P09C06 & DENV NS1 & 21 & 1 & 3 & AREEARQLGLFYAMDY & V1-14 & 4 & 16 & 0.082 & 4 & 1.017 & 0.014 & 0 \\
\hline P09B08 & DENV NS1 & 21 & 1 & 6 & ARLDFIRYGSSYEAMDY & V5-9-2 & 4 & 17 & 0.08 & 3 & 0.655 & 0.013 & 0 \\
\hline P09B10 & DENV NS1 & 21 & 1 & 2 & ARHYAMDY & V5-12 & 4 & 8 & -0.137 & 1 & 0.549 & 0.011 & 0 \\
\hline P09C02 & DENV NS1 & 21 & 1 & 3 & ARTGYDYDSAWFAY & V5-6-5 & 3 & 14 & -0.228 & 2 & 0.674 & 0.01 & 0 \\
\hline
\end{tabular}

